\title{
Finite Discrete, Shift-Invariant, Directional Filterbanks for Visual Information Processing, I : Construction
}

\author{
Hitoshi ARAI ${ }^{1 *}$ and Shinobu ARAI \\ ${ }^{1}$ Graduate School of Mathematical Sciences, the University of Tokyo, \\ Komaba 3-8-1, Meguro-ku, Tokyo 153-8914, Japan \\ E-mail:h-arai@ms.u-tokyo.ac.jp
}

Received February 26, 2007; final version accepted July 6, 2007

\begin{abstract}
As is well known in neuroscience, simple cells of the mammalian's striate cortex possess both orientation and spatial-frequency selectivity, and are similar to the Gabor filters or Gaussian derivative filters in shape. The purpose of this paper is to propose a method of designing perfect reconstruction $2 \mathrm{D}$ filterbanks which act on finite dimensional linear spaces consisting of $2 \mathrm{D}$ signals of a certain size, and have several analogous features to simple cells: (1) the filterbanks consist of several spatial-frequency channels with orientation selectivity, (2) the filterbanks have shift-invariant multiresolution (multiscale) structures, (3) filters contained in them are FIR, and are similar in appearance to not only Gaussian derivatives of 1st and 2nd order, but also ones of higher order. Moreover, they are constructed by finite linear combinations of separable filters. As is described in the text, by virtue of these properties, our 2D filterbanks can become bases of constructing computational nonlinear models of visual information processing. In this paper we construct the 2D filterbanks, and discuss them from the viewpoint of vision science. For example we disclose a possible role of "Gaussian-derivative-like" filters of higher order in our filterbanks. Practical applications of our 2D filterbanks to vision science and image processing will be given in our subsequent papers.
\end{abstract}

KEYWORDS: filterbanks, frames, orientation selectivity, spatial-frequency selectivity, visual cortex

\section{Introduction}

After the landmark discovery by Hubel and Wiesel about receptive field characteristics of cortical cells of cats and monkeys ([13], [14], [15]), many researchers have studied the role of the cells in the striate cortex, and proposed a lot of theories and models of cortical cells. For example, firstly simple cells were regarded as "edge detector". Afterwards however Marr and Hildreth ([18]; see also Marr [19]) regarded even-symmetric simple cells as "zero-crossing detector". Somewhat later De Valois et al. found that many receptive fields of cells in macaque monkey's striate cortex behave like narrow bandpass spatial filters with orientation tuning, and at a given retinal eccentricity the distribution of peak frequency covers a large area of spatial-frequency ([8], [9]). Their findings are known to provide a hard physiological evidence supporting the multiple-channel hypothesis for the visual system ([3]). Furthermore, since experimentally observed receptive field data are accurately fitted by oriented Gabor functions ([30]) or by oriented Gaussian derivatives ([31]), so far these functions have been widely used to study modeling of vision at the visual cortex level.

In this paper, taking these developments of vision science into account, we propose a method of constructing 2D frame filters which have some mathematical advantages over oriented Gabor filters and oriented Gaussian derivative filters, although ours are similar to them in shape. More specifically, we construct 2D filterbanks so that they possess the following features (1)-(4) which are desirable for computer based study of vision:

(1) The 2D filterbank possesses filters having spatial-frequency and orientation selectivity.

(2) It is perfect reconstruction and shift-invariant, and moreover has a multiresolution (multiscale) structure.

(3) It consists of FIR filters which are similar to Gaussian derivatives in shape. (For convenience in this paper, we call the FIR filter similar to the $\mu$-th Gaussian derivative the Gaussian-derivative-like filter of order $\mu$. For its precise definition, see Section 8.)

Before stating the next feature (4), we mention briefly one of advantages of the property (3). In practical computation, Gaussian derivatives as well as Gabor functions need to be discretized and approximated, because they are not compactly supported. However FIR filters need no such approximation. Moreover from the viewpoint of vision, since sizes of receptive fields of simple cells are small, it is natural that they are modeled rather by FIR filters. (For details, see Section 8.)

(4) The 2D filterbank is produced by combining tensor products of 1D filters. Notwithstanding, it possesses as many orientation selectivity as we need.

* The first author is supported partly by the Grant-in-Aid for Scientific Research (B), Japan Society for the Promotion of Science. 
Reasons why these properties are useful for mathematical study of vision are described in Section 8 . It should be noted that, in general, the role of filters like higher derivatives of Gaussian functions in the visual system is largely unknown (see [4]). However, since our filterbank contains Gaussian-derivative-like filters of lower order and higher order, it is possible to study mathematically how they work in visual information processing. One of our conclusions is that Gaussian-derivative-like filters of lower order and higher order improve collaboratively the performance of orientation selectivity of the filterbanks. For details, see Section 8.

So far, from mathematical or engineering interest rather than applications to vision science, various types of directional 2D filterbanks were proposed by many researchers. For example, curvelets by Candes and Donoho ([5]), complex wavelets by Kingsbury ([16]), contourlets by Do and Vetterli ([10]), and other filterbanks presented in Matsuo, Yoshida and Nakamori [20], Nguyen [22], and Vandergheynst [29]. However our construction is different from them.

This paper is organized as follows. Section 2 is devoted to summarize basic mathematical terminology and notion which are used in later sections. In Sections 3, 4 and 5 we construct 2D filterbanks with the properties (1)-(4). The construction is constituted from two steps. In Section 3 we describe the first step: we construct shift-invariant 2D filterbanks by tensor products of framlet filters, and give a sufficient condition for such filterbanks to be perfect reconstruction. These filterbanks do not possess enough orientation selectivity for studying vision, however we find out that some of them have potential ability of selecting orientation. As the second step we endow them with plentiful orientation selectivity without losing the perfect reconstruction property. The procedure is explained in Sections 4 and 5. In Section 6 we show figures of impulse responses and their spectra in order to see how the 2D filterbanks can cover many orientation. In Section 7 we show examples of image processing by using a 2D filterbank proposed in this paper. In Section 8, we discuss our 2D filterbanks from viewpoint of vision science.

\section{Mathematical Glossary-Periodization, Cyclic Convolution etc.-}

In this section we describe some mathematical notion and terminology. Throughout this paper we denote by $\boldsymbol{Z}$ the set of all integers. For a positive integer $N$, let $\boldsymbol{Z}_{N}$ be the set of integers $0,1, \ldots, N-1$. Denote by $l(\boldsymbol{Z})$ the set of all arrays $(x[n])_{n \in \boldsymbol{Z}}$ of infinite complex numbers, and by $l\left(\boldsymbol{Z}_{N}\right)$ the set of all arrays of $N$ complex numbers. We write elements of $l\left(\boldsymbol{Z}_{N}\right)$ usually by boldfaces $\mathbf{x}, \mathbf{y}, \mathbf{a}, \mathbf{b}, \ldots$ Elements of $l\left(\boldsymbol{Z}_{N}\right)$ are often extended to arrays of infinite complex numbers by several ways. One way is the so-called periodic extension: for $\mathbf{x}=(x[n])_{n \in \boldsymbol{Z}_{N}}$, let $x_{\text {per }}[n+k N]=x[n]$ for all $n \in \boldsymbol{Z}_{N}$ and $k \in Z$. Then $x_{\text {per }}=\left(x_{\text {per }}[n]\right)_{n \in Z}$ is called the $N$-periodic extension of $\mathbf{x}$ or simply the periodic extension of $\mathbf{x}$. Other ways are zero-padding extension and symmetric extension which are defined as follows. For $\mathbf{x}=(x[n])_{n \in \boldsymbol{Z}_{N}}$, let $x_{\text {zero }}[n]=x[n]$ for $n \in \boldsymbol{Z}_{N}$ and $x_{\text {zero }}[n]=0$ for $n \notin \boldsymbol{Z}_{N}$, and $x_{\text {zero }}=\left(x_{\text {zero }}[n]\right)_{n \in \boldsymbol{Z}}$ is the zero-padding extension of $\mathbf{x}$. The symmetric extension of $\mathbf{x}$ is defined by $x_{\text {sym }}=\left(x_{\text {sym }}[n]\right)_{n \in \boldsymbol{Z}}$, where $x_{\text {sym }}[n]=x[n]$ for $n \in \boldsymbol{Z}_{N}$ and $x_{\text {sym }}[n+N]=$ $x[N-1-n]$ for $n \in \boldsymbol{Z}_{N}$, and $x_{\text {sym }}[n+2 k N]=x_{\text {sym }}[n]$ for all $k \in \boldsymbol{Z}$ and $n \in \boldsymbol{Z}_{2 N}$.

For a stable filter $h=(h[n])_{n \in Z}$, let us denote its frequency response by $H(\theta)$, that is

$$
H(\theta)=\sum_{n \in \boldsymbol{Z}} h[n] e^{-2 \pi i n \theta}, \quad \theta \in(-\infty, \infty)
$$

and its $N$-periodization by $p_{N}(h)$, that is

$$
p_{N}(h)[n]=\sum_{k \in \boldsymbol{Z}} h[n+k N]
$$

Let $\mathbf{p}_{N}(h)=\left(p_{N}(h)[n]\right)_{n \in Z_{N}}$.

For $\mathbf{x}=(x[n])_{n \in \boldsymbol{Z}_{N}}$ and $\mathbf{y}=(y[n])_{n \in \boldsymbol{Z}_{N}}$, the cyclic convolution $\mathbf{x} *_{N} \mathbf{y}=\left(x *_{N} y[n]\right)_{n \in \boldsymbol{Z}_{N}}$ of $\mathbf{x}$ and $\mathbf{y}$ is defined by

$$
x *_{N} y[n]=\sum_{m=0}^{N-1} x_{p e r}[n-m] y[m] .
$$

For mathematical convenience, let

$$
x \star_{N} y[n]=\sum_{m=0}^{N-1} x_{p e r}[n+m] y[m],
$$

and $\mathbf{x} \star_{N} \mathbf{y}=\left(x \star_{N} y[n]\right)_{n \in \boldsymbol{Z}_{N}}$.

As usual, for $\mathbf{x}=(x[n])_{n \in Z_{N}}$, its DFT $\mathcal{F}_{N}(\mathbf{x})=\left(\mathcal{F}_{N}(\mathbf{x})[n]\right)_{n \in Z_{N}}$ is defined by

$$
\mathcal{F}_{N}(\mathbf{x})[n]=\sum_{m=0}^{N-1} x[m] \exp \left(-2 \pi i n \frac{m}{N}\right),
$$

and we denote IDFT of $\mathbf{x}$ by $\mathcal{F}_{N}^{-1}(\mathbf{x})=\left(\mathcal{F}_{N}^{-1}(\mathbf{x})[n]\right)_{n \in Z_{N}}$.

The terminology is extended to the case of 2-dimensional arrays. Suppose $N_{1}$ and $N_{2}$ are positive integers. Let $l\left(\boldsymbol{Z}_{N_{1}} \times \boldsymbol{Z}_{N_{2}}\right)$ be the set of all 2-dimensional array 


$$
\mathbf{x}=\left(x\left[n_{1}, n_{2}\right]\right)_{n_{1} \in \boldsymbol{Z}_{N_{1}}, n_{2} \in \boldsymbol{Z}_{N_{2}}}=\left(\begin{array}{ccc}
x[0,0] & \cdots & x\left[0, N_{2}-1\right] \\
\vdots & \ddots & \vdots \\
x\left[N_{1}-1,0\right] & \cdots & x\left[N_{1}-1, N_{2}-1\right]
\end{array}\right)
$$

of $N_{1} N_{2}$ complex numbers. For $\mathbf{x}=\left(x\left[n_{1}, n_{2}\right]\right)_{n_{1} \in \boldsymbol{Z}_{N_{1}}, n_{2} \in \boldsymbol{Z}_{N_{2}}}$, its periodic extension, zero-padding and symmetric extension are defined as follows. Let

$$
x_{p e r}\left[n_{1}+k_{1} N_{1}, n_{2}+k_{2} N_{2}\right]=x\left[n_{1}, n_{2}\right]
$$

for $n_{j} \in \boldsymbol{Z}_{N_{j}}, k_{j} \in \boldsymbol{Z}, j=1,2$. Then $x_{\text {per }}=\left(x_{\text {per }}\left[n_{1}, n_{2}\right]\right)_{n_{1} \in \boldsymbol{Z}, n_{2} \in \boldsymbol{Z}}$ is called the $N_{1} \times N_{2}$-periodic extension of $\mathbf{x}$ or the simply the periodic extension of $\mathbf{x}$. Let $x_{\text {zero }}\left[n_{1}, n_{2}\right]=x\left[n_{1}, n_{2}\right]$ for $n_{j} \in \boldsymbol{Z}_{N_{j}}, j=1,2$, and $x_{\text {zero }}\left[n_{1}, n_{2}\right]=0$ for $\left(n_{1}, n_{2}\right) \notin \boldsymbol{Z}_{N_{1}} \times \boldsymbol{Z}_{N_{2}}$. The array $x_{\text {zero }}=\left(x_{\text {zero }}\left[n_{1}, n_{2}\right]\right)_{n_{1} \in Z, n_{2} \in \boldsymbol{Z}}$ is the zero-padding extension of $\mathbf{x}$. For $\left(n_{1}, n_{2}\right) \in$ $\boldsymbol{Z}_{N_{1}} \times \boldsymbol{Z}_{N_{2}}$, let $x_{s y m}\left[n_{1}, n_{2}\right]=x\left[n_{1}, n_{2}\right]$ and

$$
\begin{aligned}
x_{\text {sym }}\left[n_{1}, n_{2}+N_{2}\right] & =x\left[n_{1}, N_{2}-1-n_{2}\right], \\
x_{\text {sym }}\left[n_{1}+N_{1}, n_{2}\right] & =x\left[N_{1}-1-n_{1}, n_{2}\right], \\
x_{\text {sym }}\left[n_{1}+N_{1}, n_{2}+N_{2}\right] & =x\left[N_{1}-1-n_{1}, N_{2}-1-n_{2}\right] .
\end{aligned}
$$

Further let $x_{\text {sym }}=\left(x_{\text {sym }}\left[n_{1}, n_{2}\right]\right)_{n_{1} \in \boldsymbol{Z}, n_{2} \in \boldsymbol{Z}}$ be the $2 N_{1} \times 2 N_{2}$-priodic extension of $\left(x_{s y m}\left[n_{1}, n_{2}\right]\right)_{n_{1} \in \boldsymbol{Z}_{2 N_{1}}, n_{2} \in \boldsymbol{Z}_{2 N_{2}}}$. In this paper we say $x_{\text {sym }}$ the symmetric extension of $\mathbf{x}$.

For $\mathbf{x} \in l\left(\boldsymbol{Z}_{N_{1}} \times \boldsymbol{Z}_{N_{2}}\right)$ and $k \in \boldsymbol{Z}$, let

$$
\begin{aligned}
\tau_{k}^{1}(\mathbf{x})\left[n_{1}, n_{2}\right] & =x_{\text {per }}\left[n_{1}-k, n_{2}\right], \\
\tau_{k}^{2}(\mathbf{x})\left[n_{1}, n_{2}\right] & =x_{\text {per }}\left[n_{1}, n_{2}-k\right],
\end{aligned}
$$

and $\tau_{k}^{l}(\mathbf{x})\left[n_{1}, n_{2}\right]=\left(\tau_{k}^{l}(\mathbf{x})\left[n_{1}, n_{2}\right]\right)_{n_{1} \in \boldsymbol{Z}_{N_{1}}, n_{2} \in \boldsymbol{Z}_{N_{2}}}, \quad l=1,2$. In particular, when $N_{1}$ and $N_{2}$ are even, let $\tau(\mathbf{x})=$ $\tau_{N_{1} / 2}^{1}\left(\tau_{N_{2} / 2}^{2}(\mathbf{x})\right)$, and let $\tau^{-1}$ the inverse map of $\tau$.

Throughout this paper we denote the 2-dimensional DFT for elements of $l\left(\boldsymbol{Z}_{N_{1}} \times \boldsymbol{Z}_{N_{2}}\right)$ by $\mathcal{F}_{N_{1}, N_{2}}$, and 2-dimensional IDFT by $\mathcal{F}_{N_{1}, N_{2}}^{-1}$. The cyclic convolution

$$
\mathbf{x} *_{N_{1}, N_{2}} \mathbf{y}=\left(x *_{N_{1}, N_{2}} y\left[n_{1}, n_{2}\right]\right)_{n_{1} \in \boldsymbol{Z}_{N_{1}}, n_{2} \in \boldsymbol{Z}_{N_{2}}}
$$

for $\mathbf{x}=\left(x\left[n_{1}, n_{2}\right]\right)_{n_{1} \in \boldsymbol{Z}_{N_{1}}, n_{2} \in \boldsymbol{Z}_{N_{2}}}$ and $\mathbf{y}=\left(y\left[n_{1}, n_{2}\right]\right)_{n_{1} \in \boldsymbol{Z}_{N_{1}}, n_{2} \in \boldsymbol{Z}_{N_{2}}}$ is defined by

$$
x *_{N_{1}, N_{2}} y\left[n_{1}, n_{2}\right]=\sum_{m_{1}=0}^{N_{1}-1} \sum_{m_{2}=0}^{N_{2}-1} x_{p e r}\left[n_{1}-m_{1}, n_{2}-m_{2}\right] y\left[m_{1}, m_{2}\right] .
$$

In addition, let

$$
x \star_{N_{1}, N_{2}} y\left[n_{1}, n_{2}\right]=\sum_{m_{1}=0}^{N_{1}-1} \sum_{m_{2}=0}^{N_{2}-1} x_{p e r}\left[n_{1}+m_{1}, n_{2}+m_{2}\right] y\left[m_{1}, m_{2}\right],
$$

and $\mathbf{x} \star_{N_{1}, N_{2}} \mathbf{y}=\left(x \star_{N_{1}, N_{2}} y\left[n_{1}, n_{2}\right]\right)_{n_{1} \in \boldsymbol{Z}_{N_{1}}, n_{2} \in \boldsymbol{Z}_{N_{2}}}$.

For $\mathbf{a}=\left(a\left[n_{1}\right]\right)_{n_{1} \in \boldsymbol{Z}_{N_{1}}}$ and $\mathbf{x}=\left(x\left[n_{1}, n_{2}\right]\right)_{n_{1} \in \boldsymbol{Z}_{N_{1}}, n_{2} \in \boldsymbol{Z}_{N_{2}}}$, let us define

$$
\mathbf{a} *_{N_{1}} \mathbf{x}=\left(a *_{N_{1}} x\left[n_{1}, n_{2}\right]\right)_{n_{1} \in \boldsymbol{Z}_{N_{1}}, n_{2} \in \boldsymbol{Z}_{N_{2}}}
$$

and $\mathbf{a} \star_{N_{1}} \mathbf{x}=\left(a \star_{N_{1}} x\left[n_{1}, n_{2}\right]\right)_{n_{1} \in \boldsymbol{Z}_{N_{1}}, n_{2} \in \boldsymbol{Z}_{N_{2}}}$ by

$$
\begin{aligned}
& a *_{N_{1}} x\left[n_{1}, n_{2}\right]=\sum_{m_{1}=1}^{N_{1}-1} a\left[n_{1}-m_{1}\right] x\left[m_{1}, n_{2}\right], \\
& a \star_{N_{1}} x\left[n_{1}, n_{2}\right]=\sum_{m_{1}=1}^{N_{1}-1} a\left[n_{1}+m_{1}\right] x\left[m_{1}, n_{2}\right],
\end{aligned}
$$

respectively. By the same way we define $\mathbf{a} *_{N_{2}} \mathbf{x}$ and $\mathbf{a} \star_{N_{2}} \mathbf{x}$ for $\mathbf{a} \in l\left(\boldsymbol{Z}_{N_{2}}\right)$.

We close the glossary with recalling "tensor product". Let $\mathbf{a}=(a[n])_{n \in \boldsymbol{Z}_{N_{1}}}$ and $\mathbf{b}=(b[n])_{n \in \boldsymbol{Z}_{N_{2}}}$. Then the tensor product of $\mathbf{a}$ and $\mathbf{b}$ is defined by

$$
\mathbf{a} \otimes \mathbf{b}=\left(a\left[n_{1}\right] b\left[n_{2}\right]\right)_{n_{1} \in \boldsymbol{Z}_{N_{1}}, n_{2} \in \boldsymbol{Z}_{N_{2}}} \in l\left(\boldsymbol{Z}_{N_{1}} \times \boldsymbol{Z}_{N_{2}}\right) .
$$




\section{Separable, Periodic, Shift-Invariant Filterbanks}

Our purpose is to construct directional shift-invariant 2D filterbanks for $l\left(\boldsymbol{Z}_{N_{1}} \times \boldsymbol{Z}_{N_{2}}\right)$. As preliminaries to the construction, in this section we are concerned with constructing a separable, periodic, shift-invariant framelet filterbank for $l\left(\boldsymbol{Z}_{N_{1}} \times \boldsymbol{Z}_{N_{2}}\right)$ from 1D stable filters. Since these filterbanks do not possess enough orientation selectivity, in the next section we make 2D filterbanks with good orientation selectivity from the filterbanks described in this section. As was pointed out in the previous paper by the first author [1], the shift-invariant filterbanks are desirable experimentally more than maximal decimated ones for studying nonlinear models of vision. Hence we intend to give 2D filterbanks this property. Originally shift-invariant wavelets were studied by Coifman and Donoho [6], Nason and Silverman [21], and Percival and Walden [25]. We use their idea in order to bring the shift-invariance to our filterbank.

In this section we assume that $N_{1}$ and $N_{2}$ are positive even numbers. The general case will be discussed in Section 9. Let $g^{(\mu)}=\left(g^{(\mu)}[n]\right)_{n \in \boldsymbol{Z}}$ and $\widetilde{g}^{(\mu)}=\left(\widetilde{g}^{(\mu)}[n]\right)_{n \in \boldsymbol{Z}}$ be stable filters $(\mu=0,1, \ldots, m)$. A simple example of these filters is a bi-orthogonal wavelet: $g^{(0)}$ is a scaling filter, $g^{(1)}$ its wavelet filter, $\widetilde{g}^{(0)}$ a dual scaling filter and $\widetilde{g}^{(1)}$ a dual wavelet filter. However for our further purpose and applications we need rather the so-called frames (see Examples 1 and 2 described later). In any case, $g^{(\mu)}$ 's will be used as filters for an analysis bank and $\widetilde{g}^{(\mu)}$ 's for a synthesis bank. Furthermore we will suppose usually that $g^{(0)}$ and $\widetilde{g}^{(0)}$ are lowpass filters, and that $g^{(\mu)}$ and $\widetilde{g}^{(\mu)}$ for $\mu>0$ are bandpass or highpass filters.

Suppose $N$ is positive even number. For $\mathbf{x} \in l\left(\boldsymbol{Z}_{N / 2}\right)$, let $(\uparrow 2) \mathbf{x}=((\uparrow 2) x[n])_{n \in Z_{N}}$ be the usual upsampler, that is

$$
(\uparrow 2) x[n]= \begin{cases}x[n / 2], & n \in \boldsymbol{Z}_{N} \text { and } n \text { is even, } \\ 0, & n \in \boldsymbol{Z}_{N} \text { and } n \text { is odd. }\end{cases}
$$

Let us introduce a variation of this upsampler. For $\mathbf{x}=(x[n])_{n \in \boldsymbol{Z}_{N}} \in l\left(\boldsymbol{Z}_{N}\right)$, let $x^{\circ}[n]=x[n]+x[n+N / 2]\left(n \in \boldsymbol{Z}_{N / 2}\right)$ and let $\mathbf{x}^{\circ}=\left(x^{\circ}[n]\right)_{n \in \boldsymbol{Z}_{N / 2}}$. Then our variant of the usual upsampler for $\mathbf{x} \in l\left(\boldsymbol{Z}_{N}\right)$ is defined by

$$
T_{N}(\mathbf{x})=(\uparrow 2) \mathbf{x}^{\circ}
$$

A difference from the usual upsampler is that $T_{N}$ is a transform from $l\left(\boldsymbol{Z}_{N}\right)$ to $l\left(\boldsymbol{Z}_{N}\right)$. Let

$$
T_{N}^{1}=T_{N}, \text { and } T_{N}^{\gamma+1}=T_{N}^{1} T_{N}^{\gamma}, \gamma=1,2, \ldots
$$

For convenience we denote by $T_{N}^{0}$ the identity map $I$.

From the stable filters $g^{(\mu)}$ and $\widetilde{g}^{(\mu)}$ we can construct the following FIR filters of length $N_{1}$ or $N_{2}$ :

$$
\begin{array}{ll}
\mathbf{g}_{1, j}^{(\mu)}=T_{N_{1}}^{j-1}\left(\mathbf{p}_{N_{1}}\left(g^{(\mu)}\right)\right), & \tilde{\mathbf{g}}_{1, j}^{(\mu)}=T_{N_{1}}^{j-1}\left(\mathbf{p}_{N_{1}}\left(\widetilde{g}^{(\mu)}\right)\right), \\
\mathbf{g}_{2, j}^{(\mu)}=T_{N_{2}}^{j-1}\left(\mathbf{p}_{N_{2}}\left(g^{(\mu)}\right)\right), & \widetilde{\mathbf{g}}_{2, j}^{(\mu)}=T_{N_{2}}^{j-1}\left(\mathbf{p}_{N_{2}}\left(\widetilde{g}^{(\mu)}\right)\right),
\end{array}
$$

$\mu=0,1, \ldots, m$, and $j=1,2,3, \ldots$ Denote by $\overline{\mathbf{g}}_{k, j}^{(\mu)}$ the array of the complex conjugate $\left(\overline{g_{k, j}^{(\mu)}[n]}\right)_{n \in \boldsymbol{Z}_{N_{k}}}$.

Now we construct an analysis bank. For $\mathbf{x} \in l\left(\boldsymbol{Z}_{N_{1}} \times \boldsymbol{Z}_{N_{2}}\right)$, let

$$
f_{j}^{\mu_{1}, \mu_{2}}(\mathbf{x})=\overline{\mathbf{g}}_{2, j}^{\left(\mu_{2}\right)} \star_{N_{2}}\left(\overline{\mathbf{g}}_{1, j}^{\left(\mu_{1}\right)} \star_{N_{1}} \mathbf{x}\right),
$$

$\mu_{1}, \mu_{2}=0,1, \ldots, m$, and $j=1,2, \ldots$ Suppose an array $\mathbf{x} \in l\left(\boldsymbol{Z}_{N_{1}} \times \boldsymbol{Z}_{N_{2}}\right)$ is given. Then we decompose it as follows:

$$
\mathbf{F}_{1}\left(\mu_{1}, \mu_{2}\right)\left(=\mathbf{F}_{1}\left(\mu_{1}, \mu_{2}, \mathbf{x}\right)\right)=f_{1}^{\mu_{1}, \mu_{2}}(\mathbf{x}), \quad \mu_{1}, \mu_{2}=0,1, \ldots, m .
$$

This decomposition is the 1 st stage of the analysis phase. The subband signal $\mathbf{F}_{1}(0,0)$ is called the 1 st level approximation coefficients of $\mathbf{x}$, and $\mathbf{F}_{1}\left(\mu_{1}, \mu_{2}\right)$ for $\mu_{1}+\mu_{2} \neq 0$ the 1 st level detail coefficients of $\mathbf{x}$. The $2 \mathrm{nd}$ stage consists of the following decomposition of the 1st level approximation coefficients of $\mathbf{x}$ :

$$
\mathbf{F}_{2}\left(\mu_{1}, \mu_{2}\right)\left(=\mathbf{F}_{2}\left(\mu_{1}, \mu_{2}, \mathbf{x}\right)\right)=f_{2}^{\mu_{1}, \mu_{2}}\left(\mathbf{F}_{1}(0,0)\right), \quad \mu_{1}, \mu_{2}=0,1, \ldots, m .
$$

Here $\mathbf{F}_{2}(0,0)$ is called the 2 nd level approximation coefficients of $\mathbf{x}$, and $\mathbf{F}_{2}\left(\mu_{1}, \mu_{2}\right)$ for $\mu_{1}+\mu_{2} \neq 0$ the 2 nd level detail coefficients of $\mathbf{x}$. In general, $j+1$ th level approximation coefficients $\mathbf{F}_{j+1}(0,0)$ and $j+1$ th level detail coefficients $\mathbf{F}_{j+1}\left(\mu_{1}, \mu_{2}\right)\left(\mu_{1}+\mu_{2} \neq 0\right)$ are defined from $\mathbf{F}_{j}(0,0)$ as follows:

$$
\mathbf{F}_{j+1}\left(\mu_{1}, \mu_{2}\right)\left(=\mathbf{F}_{j+1}\left(\mu_{1}, \mu_{2}, \mathbf{x}\right)\right)=f_{j+1}^{\mu_{1}, \mu_{2}}\left(\mathbf{F}_{j}(0,0)\right), \quad \mu_{1}, \mu_{2}=0,1, \ldots, m .
$$

The analysis bank is depicted as Fig. 1.

Next we consider the synthesis bank. For $\mathbf{x} \in l\left(\boldsymbol{Z}_{N_{1}} \times \boldsymbol{Z}_{N_{2}}\right)$, let

$$
\widetilde{f}_{j}^{\mu_{1}, \mu_{2}}(\mathbf{x})=\widetilde{\mathbf{g}}_{2, j}^{\left(\mu_{2}\right)} *_{N_{2}}\left(\widetilde{\mathbf{g}}_{1, j}^{\left(\mu_{1}\right)} *_{N_{1}} \mathbf{x}\right) .
$$

The filterbank indicated by Fig. 2 is a synthesis bank for this analysis bank.

Let 


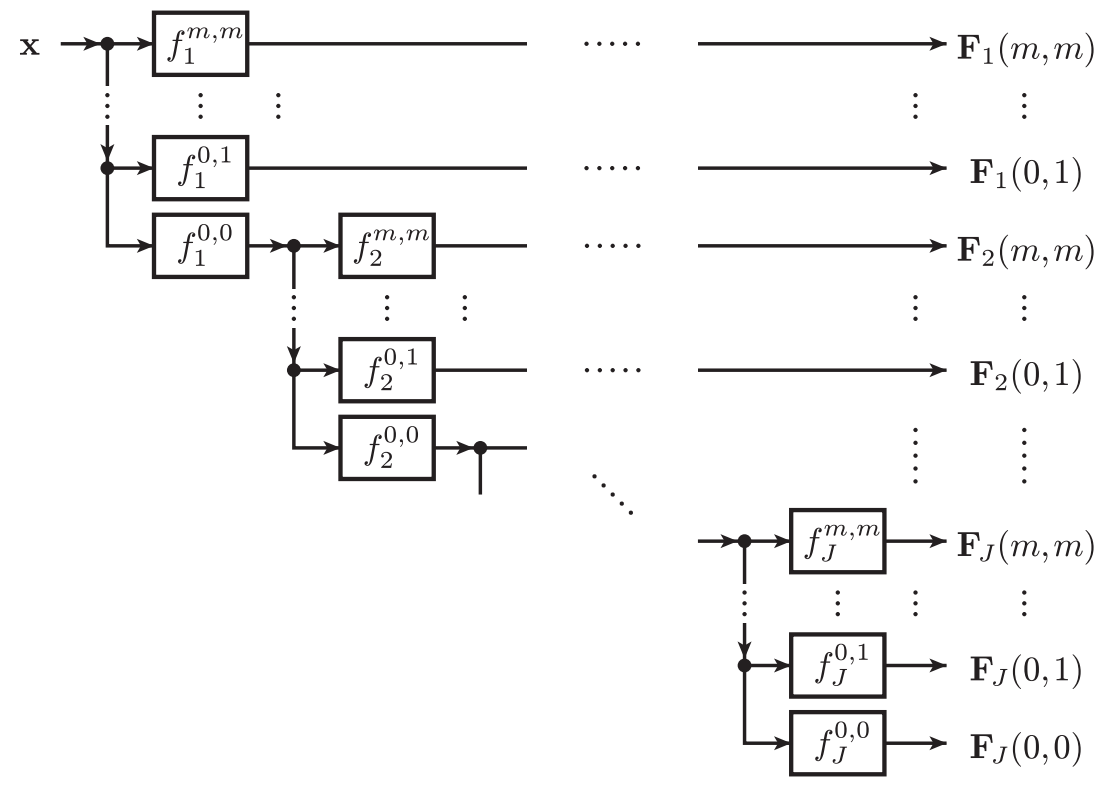

Fig. 1.

$$
\begin{aligned}
\widetilde{\mathbf{F}}_{J}\left(\mu_{1}, \mu_{2}\right)\left(=\widetilde{\mathbf{F}}_{J}\left(\mu_{1}, \mu_{2}, \mathbf{x}\right)\right) & =\frac{1}{4^{J}} \widetilde{f}_{1}^{0,0}\left(\cdots \widetilde{f}_{J-1}^{0,0}\left(\widetilde{f}_{J}^{\mu_{1}, \mu_{2}}\left(\mathbf{F}_{J}\left(\mu_{1}, \mu_{2}\right)\right)\right) \cdots\right), \\
\widetilde{\mathbf{F}}_{J-1}\left(\mu_{1}, \mu_{2}\right)\left(=\widetilde{\mathbf{F}}_{J-1}\left(\mu_{1}, \mu_{2}, \mathbf{x}\right)\right) & =\frac{1}{4^{J-1}} \widetilde{f}_{1}^{0,0}\left(\cdots \widetilde{f}_{J-2}^{0,0}\left(\widetilde{f}_{J-1}^{\mu_{1}, \mu_{2}}\left(\mathbf{F}_{J-1}\left(\mu_{1}, \mu_{2}\right)\right)\right) \cdots\right), \\
\vdots & \\
\widetilde{\mathbf{F}}_{1}\left(\mu_{1}, \mu_{2}\right)\left(=\widetilde{\mathbf{F}}_{1}\left(\mu_{1}, \mu_{2}, \mathbf{x}\right)\right) & =\frac{1}{4} \widetilde{f}_{1}^{\mu_{1}, \mu_{2}}\left(\mathbf{F}_{1}\left(\mu_{1}, \mu_{2}\right)\right) .
\end{aligned}
$$

Then the filterbank defined by combining the analysis bank indicated by Fig. 1 and the synthesis bank by Fig. 2 is equivalent to the filterbank defined by Fig. 3.

If the filterbank is perfect reconstruction (PR), that is, for any $\mathbf{x} \in l\left(\boldsymbol{Z}_{N_{1}} \times \boldsymbol{Z}_{N_{2}}\right)$,

$$
\mathbf{x}=\widetilde{\mathbf{F}}_{J}(0,0)+\sum_{j=1}^{J} \sum_{\substack{\mu_{1}, \mu_{2}=0 \\ \mu_{1}+\mu_{2} \neq 0}}^{m} \widetilde{\mathbf{F}}_{j}\left(\mu_{1}, \mu_{2}\right),
$$
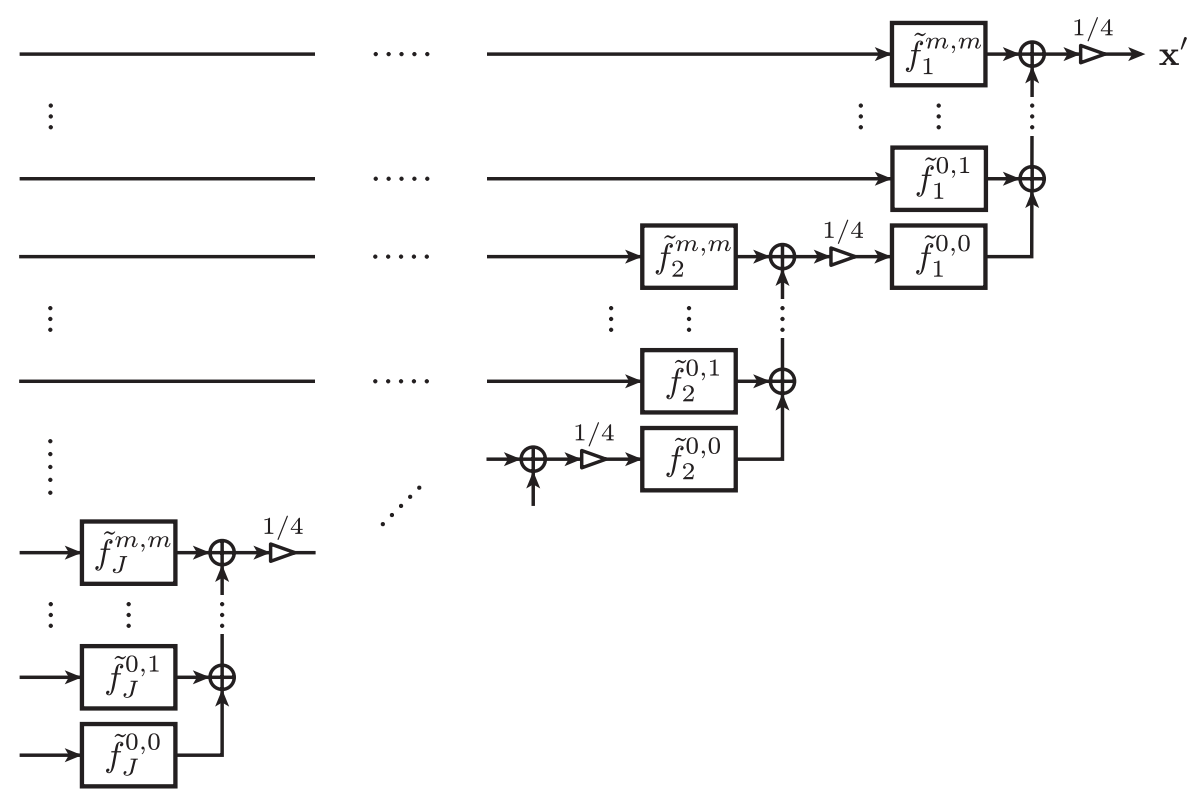

Fig. 2. 


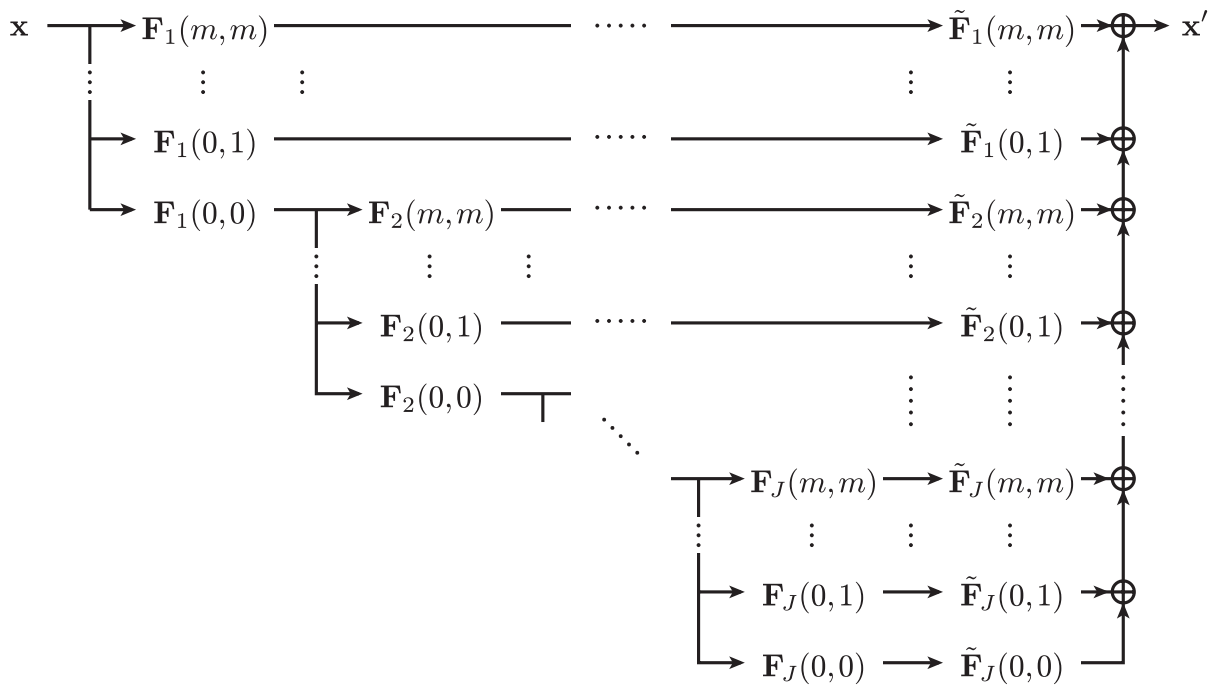

Fig. 3.

then we say that the family of filters $\left\{g^{(0)}, \ldots, g^{(m)} ; \widetilde{g}^{(0)}, \ldots, \widetilde{\boldsymbol{g}}^{(m)}\right\}$ is a finite, perfect reconstruction, maximal overlap filterbank (abbr. FPRMO filterbank).

We have the following sufficient condition for the filterbank to be FPRMO filterbank.

Theorem 1. Suppose $G^{(\mu)}$ and $\widetilde{G}^{(\mu)}$ are the frequency responses of $g^{(\mu)}$ and $\widetilde{g}^{(\mu)}$ respectively. If

$$
\sum_{\mu=0}^{m} \widetilde{G}^{(\mu)}(\theta) \overline{G^{(\mu)}(\theta)}=2, \quad \text { for all } \theta \in[0,1),
$$

then $\left\{g^{(0)}, \ldots, g^{(m)} ; \widetilde{\boldsymbol{g}}^{(0)}, \ldots, \widetilde{\boldsymbol{g}}^{(m)}\right\}$ is an FPRMO filterbank.

To prove this theorem we begin with demonstrating the following lemma.

Lemma 2. Suppose $N$ is a positive even number. Let $x=(x[n])_{n \in Z}$ be a stable filter. Then for $v=1,2, \ldots$,

$$
\mathcal{F}_{N}\left(T_{N}^{v}\left(\mathbf{p}_{N}(x)\right)\right)[k]=X\left(\frac{2^{v} k}{N}\right), \text { for all } k \in Z_{N} .
$$

Proof. Note that

$$
\begin{aligned}
\mathcal{F}_{N}\left(\mathbf{p}_{N}(x)\right)[k] & =\sum_{n=0}^{N-1}\left(\sum_{s \in \boldsymbol{Z}} x[n+s N]\right) \exp \left(-2 \pi i k \frac{n}{N}\right) \\
& =\sum_{n=0}^{N-1}\left(\sum_{s \in \boldsymbol{Z}} x[n+s N]\right) \exp \left(-2 \pi i k \frac{n+s N}{N}\right) \\
& =\sum_{m \in \boldsymbol{Z}} x[m] \exp \left(-2 \pi i k \frac{m}{N}\right)=X\left(\frac{k}{N}\right)
\end{aligned}
$$

Let $M=N / 2$. First we prove the lemma when $v=1$. For $\mathbf{y}=(y[n])_{n \in \boldsymbol{Z}_{N}} \in l\left(\boldsymbol{Z}_{N}\right)$, we have

$$
\begin{aligned}
\mathcal{F}_{M}\left(\mathbf{y}^{\circ}\right)[k] & =\sum_{n=0}^{M-1}(y[n]+y[n+M]) \exp \left(-2 \pi i k \frac{n}{M}\right) \\
& =\sum_{n=0}^{M-1} y[n] \exp \left(-2 \pi i(2 k) \frac{n}{N}\right)+\sum_{n=M}^{N-1} y[n] \exp \left(-2 \pi i(2 k) \frac{n}{N}\right) \\
& =\mathcal{F}_{N}(\mathbf{y})[2 k] .
\end{aligned}
$$

Hence

$$
\begin{aligned}
\mathcal{F}_{N}\left(T_{N}(\mathbf{y})\right)[k] & =\sum_{n=0}^{M-1} y^{\circ}[n] \exp \left(-2 \pi i k \frac{2 n}{N}\right)=\mathcal{F}_{M}\left(\mathbf{y}^{\circ}\right)[k] \\
& =\mathcal{F}_{N}(\mathbf{y})[2 k] .
\end{aligned}
$$




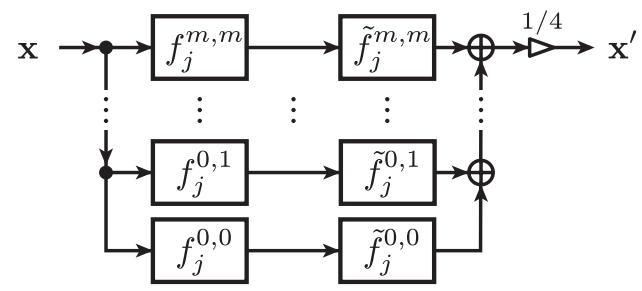

Fig. 4.

Therefore

$$
\mathcal{F}_{N}\left(T_{N}\left(\mathbf{p}_{N}(x)\right)\right)[k]=\mathcal{F}_{N}\left(\mathbf{p}_{N}(x)\right)[2 k]=X\left(\frac{2 k}{N}\right) .
$$

Next we prove the general case. If the lemma holds true for $v-1$, then by this hypothesis and (3) we have that

$$
\mathcal{F}_{N}\left(T_{N}^{\nu}\left(\mathbf{p}_{N}(x)\right)\right)[k]=\mathcal{F}_{N}\left(T_{N}^{\nu-1}\left(\mathbf{p}_{N}(x)\right)\right)[2 k]=X\left(\frac{2^{v} k}{N}\right)
$$

Now we turn to prove Theorem 1. For $\mathbf{x}=(x[n])_{n \in \boldsymbol{Z}_{N}}$ and $\mathbf{y}=(y[n])_{n \in \boldsymbol{Z}_{N}}$, let $\mathbf{x}_{\bullet} \mathbf{y}=(x[n] y[n])_{n \in \boldsymbol{Z}_{N}}$.

Proof of Theorem 1. It is enough to prove that for each $j$ the filterbank (Fig. 4) is PR.

By the definition of $\mathbf{x}^{\prime}$ in Fig. 4, we have

$$
\mathbf{x}^{\prime}=\frac{1}{4} \sum_{\mu_{1}, \mu_{2}=0}^{m} \tilde{\mathbf{g}}_{2, j}^{\left(\mu_{2}\right)} *_{N_{2}}\left(\widetilde{\mathbf{g}}_{1, j}^{\left(\mu_{1}\right)} *_{N_{1}}\left(\overline{\mathbf{g}}_{2, j}^{\left(\mu_{2}\right)} \star_{N_{2}}\left(\overline{\mathbf{g}}_{1, j}^{\left(\mu_{1}\right)} \star_{N_{1}} \mathbf{x}\right)\right)\right) .
$$

Therefore what we need to prove is the following

$$
\mathbf{x}=\frac{1}{4} \sum_{\mu_{1}, \mu_{2}=0}^{m} \widetilde{\mathbf{g}}_{2, j}^{\left(\mu_{2}\right)} *_{N_{2}}\left(\widetilde{\mathbf{g}}_{1, j}^{\left(\mu_{1}\right)} *_{N_{1}}\left(\overline{\mathbf{g}}_{2, j}^{\left(\mu_{2}\right)} \star_{N_{2}}\left(\overline{\mathbf{g}}_{1, j}^{\left(\mu_{1}\right)} \star_{N_{1}} \mathbf{x}\right)\right)\right) .
$$

Since $\mathbf{x}$ is represented by a finite linear combination of the tensor products of 1 dimensional arrays, it is sufficient to prove the equation (4) when $\mathbf{x}=\mathbf{a}_{1} \otimes \mathbf{a}_{2}$ for some $\mathbf{a}_{1} \in l\left(\boldsymbol{Z}_{N_{1}}\right)$ and $\mathbf{a}_{2} \in l\left(\boldsymbol{Z}_{N_{2}}\right)$. In this case we have

$$
\begin{aligned}
\mathbf{x}^{\prime} & =\frac{1}{4} \sum_{\mu_{1}, \mu_{2}=0}^{m} \widetilde{\mathbf{g}}_{2, j}^{\left(\mu_{2}\right)} *_{N_{2}}\left(\widetilde{\mathbf{g}}_{1, j}^{\left(\mu_{1}\right)} *_{N_{1}}\left(\overline{\mathbf{g}}_{2, j}^{\left(\mu_{2}\right)} \star_{N_{2}}\left(\overline{\mathbf{g}}_{1, j}^{\left(\mu_{1}\right)} \star_{N_{1}} \mathbf{a}_{1} \otimes \mathbf{a}_{2}\right)\right)\right) \\
& =\frac{1}{2} \sum_{\mu_{1}=0}^{m}\left(\widetilde{\mathbf{g}}_{1, j}^{\left(\mu_{1}\right)} *_{N_{1}}\left(\overline{\mathbf{g}}_{1, j}^{\left(\mu_{1}\right)} \star_{N_{1}} \mathbf{a}_{1}\right)\right) \otimes \frac{1}{2} \sum_{\mu_{2}=0}^{m}\left(\widetilde{\mathbf{g}}_{2, j}^{\left(\mu_{2}\right)} *_{N_{2}}\left(\overline{\mathbf{g}}_{2, j}^{\left(\mu_{2}\right)} \star_{N_{2}} \mathbf{a}_{2}\right)\right) .
\end{aligned}
$$

Consequently it is enough to prove that

$$
\frac{1}{2} \sum_{\mu=0}^{m}\left(\widetilde{\mathbf{g}}_{l, j}^{(\mu)} *_{N_{l}}\left(\overline{\mathbf{g}}_{l, j}^{(\mu)} \star_{N_{l}} \mathbf{a}_{l}\right)\right)=\mathbf{a}_{l}
$$

for $l=1,2$. Let $\mathbf{a}_{l}^{\prime}$ be the left hand side of (5). Then

$$
\mathcal{F}_{N_{l}}\left(\mathbf{a}_{l}^{\prime}\right)=\frac{1}{2} \sum_{\mu=0}^{m} \mathcal{F}_{N_{l}}\left(\widetilde{\mathbf{g}}_{l, j}^{(\mu)}\right) \overline{\mathcal{F}_{N_{l}}\left(\mathbf{g}_{l, j}^{(\mu)}\right)} \mathcal{F}_{N_{l}}\left(\mathbf{a}_{l}\right) .
$$

Therefore $\mathbf{a}_{l}=\mathbf{a}_{l}^{\prime}$ if the filters $\widetilde{\mathbf{g}}_{l, j}^{(\mu)}$ and $\mathbf{g}_{l, j}^{(\mu)}$ satisfy

$$
\mathbf{1}_{N_{l}}=\frac{1}{2} \sum_{\mu=0}^{m} \widetilde{F}_{N_{l}}\left(\widetilde{\mathbf{g}}_{l, j}^{(\mu)}\right) \overline{\mathcal{F}_{N_{l}}\left(\mathbf{g}_{l, j}^{(\mu)}\right)}
$$

where $\mathbf{1}_{N_{l}}=(1,1, \ldots, 1) \in l\left(\boldsymbol{Z}_{N_{l}}\right)$. Since $\mathbf{g}_{l, j}^{(\mu)}=T_{N_{l}}^{j-1}\left(\mathbf{p}_{N_{l}}\left(g^{(\mu)}\right)\right)$ and $\widetilde{\mathbf{g}}_{l, j}^{(\mu)}=T_{N_{l}}^{j-1}\left(\mathbf{p}_{N_{l}}\left(\widetilde{\boldsymbol{g}}^{(\mu)}\right)\right)$, by Lemma 2 we obtain (6) from (2).

Example 1. In [26], Ron and Shen introduced the so-called unitary extension property: a family of stable filters $\left\{g^{(\mu)}\right\}_{\mu=0,1, \ldots, m}$ satisfies the unitary extension property if 


$$
\begin{aligned}
\sum_{\mu=0}^{m} G^{(\mu)}(\theta) \overline{G^{(\mu)}(\theta)} & =2, \text { and } \\
\sum_{\mu=0}^{m} G^{(\mu)}(\theta) \overline{G^{(\mu)}\left(\theta+\frac{1}{2}\right)} & =0
\end{aligned}
$$

for all $\theta \in[0,1)$. They proved that this property is sufficient for a system of functions on the real line $\boldsymbol{R}$ produced from $\left\{g^{(\mu)}\right\}_{\mu=0,1, \ldots, m}$ to be a tight frame in $L^{2}(\boldsymbol{R})$, the Hilbert space of all square integrable functions on $\boldsymbol{R}$. On the other hand, from our theorem, Theorem 1, it follows that if $\left\{g^{(\mu)}\right\}_{\mu=0,1, \ldots, m}$ satisfies (7) (see also (2) in Theorem 1), then $\left\{g^{(0)}, \ldots, g^{(m)} ; g^{(0)}, \ldots, g^{(m)}\right\}$ is an FPRMO filterbank. Consequently by Theorem 1 we have that if $\left\{g^{(\mu)}\right\}_{\mu=0,1, \ldots, m}$ satisfies the unitary extension property, then $\left\{g^{(0)}, \ldots, g^{(m)} ; g^{(0)}, \ldots, g^{(m)}\right\}$ is an FPRMO filterbank. For example, Ron and Shen showed that FIR filters $\left\{g^{(\mu)}\right\}_{\mu=0,1, \ldots, m}$ constructed by the following way satisfy the unitary extension property ([26]): $g_{m}^{(\mu)}=\left(g_{m}^{\mu}[n]\right)_{n \in \boldsymbol{Z}}$ is the inverse discrete time Fourier transform of

$$
\sqrt{2} i^{\mu} e^{\pi i r(m) \theta} \sqrt{\left(\begin{array}{l}
m \\
\mu
\end{array}\right)} \sin ^{\mu}(\pi \theta) \cos ^{m-\mu}(\pi \theta),
$$

where $m$ is a positive integer, $\mu=0,1, \ldots, m$, and

$$
r(m)= \begin{cases}1, & m \text { is odd } \\ 0, & m \text { is even }\end{cases}
$$

We will use these filters in later section.

Example 2. It should be noted that there are filters which satisfy (7) but not the unitary extension property. The following filter bank is such an example:

$$
\begin{aligned}
& g^{(0)}[n]=\left\{\begin{array}{ll}
\sqrt{2} / 3, & n=1,2,3 \\
0, & \text { otherwise }
\end{array} g^{(1)}[n]= \begin{cases}-1 / 3, & n=1,3 \\
2 / 3, & n=2 \\
0, & \text { otherwise }\end{cases} \right. \\
& g^{(2)}[n]= \begin{cases}1 / \sqrt{3}, & n=1 \\
-1 / \sqrt{3}, & n=3 \\
0, & \text { otherwise }\end{cases}
\end{aligned}
$$

Indeed we have

$$
\begin{aligned}
\sum_{\mu=0}^{2} G^{(\mu)}(\theta) \overline{G^{(\mu)}(\theta)} & =2, \text { and } \\
\sum_{\mu=0}^{2} G^{(\mu)}(\theta) \overline{G^{(\mu)}\left(\theta+\frac{1}{2}\right)} & =-\frac{2}{3} \neq 0 .
\end{aligned}
$$

Therefore $\left\{g^{(0)}, g^{(1)}, g^{(2)} ; g^{(0)}, g^{(1)}, g^{(2)}\right\}$ is an FPRMO filterbank while it does not satisfy the unitary extension property. Furthermore, it is easy to see that $\left\{g^{(\mu)}\right\}_{\mu=0,1,2}$ does not satisfy also the oblique extension property introduced in [7].

\section{A DFT Construction of a Shift-Invariant Directional Filterbank}

In this section we construct 2D perfect reconstruction filterbanks which have many orientation selectivity. Throughout this section we assume that $N_{1}$ and $N_{2}$ are positive even integers, and that $g^{(\mu)}=\left(g^{(\mu)}[n]\right)_{n \in Z}$, $\widetilde{\boldsymbol{g}}^{(\mu)}=\left(\widetilde{\boldsymbol{g}}^{(\mu)}[n]\right)_{n \in \boldsymbol{Z}}, \mu=0,1, \ldots, m$ are filters satisfying the condition (2) in Theorem 1 . Note that if $g^{(0)}$ is a scaling filter, $g^{(1)}$ a corresponding bi-orthogonal (or orthogonal) wavelet filter, and $\widetilde{g}^{(0)}, \widetilde{g}^{(1)}$ are their duals -in this case $m$ must be $1-$, then for a given image $\mathbf{x}, \mathbf{F}_{j}(0,1)$ and $\mathbf{F}_{j}(1,0)$ extract vertical and horizontal components of $\mathbf{x}$ respectively, and $\mathbf{F}_{j}(1,1)$ contains its diagonal component. In general case, for example filters in Examples 1 or 2 , we can observe that $\mathbf{F}_{j}\left(0, \mu_{2}\right)$ and $\mathbf{F}_{j}\left(\mu_{1}, 0\right), \mu_{1}, \mu_{2}>0$, filter out vertical and horizontal parts of $\mathbf{x}$ respectively, and when $\mu_{1} \mu_{2}>0$, $\mathbf{F}_{j}\left(\mu_{1}, \mu_{2}\right)$ includes a certain diagonal component. However there is a remarkable difference between wavelets and such filters as in Examples 1 and 2. In the latter case, $\mathbf{F}_{j}\left(\mu_{1}, \mu_{2}\right), \mu_{1} \mu_{2}>0$, contain different diagonal components in angles of aperture. Therefore if we can decompose $\mathbf{F}_{j}\left(\mu_{1}, \mu_{2}\right), \mu_{1} \mu_{2}>0$, into opposite components in orientation, we can gain filterbanks with good orientation selectivity. We begin this section with giving filters which can separate $\mathbf{F}_{j}\left(\mu_{1}, \mu_{2}\right)$ for $\mu_{1} \mu_{2}>0$ into such two parts. For this purpose, let us decompose the frequency domain obtained by DFT as follows: 


$$
\begin{aligned}
I_{1} & =\left\{\left(n_{1}, n_{2}\right) \in \boldsymbol{Z}_{N_{1}} \times \boldsymbol{Z}_{N_{2}}: 1 \leq n_{1} \leq \frac{N_{1}}{2}-1, \frac{N_{2}}{2}+1 \leq n_{2} \leq N_{2}-1\right\}, \\
I_{2} & =\left\{\left(n_{1}, n_{2}\right) \in \boldsymbol{Z}_{N_{1}} \times \boldsymbol{Z}_{N_{2}}: 1 \leq n_{1} \leq \frac{N_{1}}{2}-1,1 \leq n_{2} \leq \frac{N_{2}}{2}-1\right\}, \\
I_{3} & =\left\{\left(n_{1}, n_{2}\right) \in \boldsymbol{Z}_{N_{1}} \times \boldsymbol{Z}_{N_{2}}: \frac{N_{1}}{2}+1 \leq n_{1} \leq N_{1}-1,1 \leq n_{2} \leq \frac{N_{2}}{2}-1\right\}, \\
I_{4} & =\left\{\left(n_{1}, n_{2}\right) \in \boldsymbol{Z}_{N_{1}} \times \boldsymbol{Z}_{N_{2}}: \frac{N_{1}}{2}+1 \leq n_{1} \leq N_{1}-1, \frac{N_{2}}{2}+1 \leq n_{2} \leq N_{2}-1\right\}, \text { and } \\
\Delta & =\left\{\left(0, n_{2}\right): n_{2} \in \boldsymbol{Z}_{N_{2}}\right\} \cup\left\{\left(\frac{N_{1}}{2}, n_{2}\right): n_{2} \in \boldsymbol{Z}_{N_{2}}\right\} \\
& \cup\left\{\left(n_{1}, 0\right): n_{1} \in \boldsymbol{Z}_{N_{1}}\right\} \cup\left\{\left(n_{1}, \frac{N_{2}}{2}\right): n_{1} \in \boldsymbol{Z}_{N_{1}}\right\} .
\end{aligned}
$$

The following is a simplified drawing of these subdomains:

$$
\begin{aligned}
& \circ 0000000 \\
& \circ * * * \quad * \quad \circ \quad * * * * \\
& \circ \quad * I_{2} \quad * \quad \circ \quad * \quad I_{1} *
\end{aligned}
$$

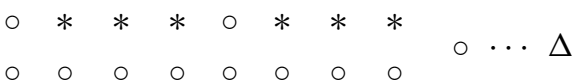

$$
\begin{aligned}
& \circ * * * \quad * \quad \circ \quad * \quad * \quad * \\
& \circ \quad * \quad I_{3} * \circ \quad \circ \quad * \quad I_{4} * \\
& \text { ○ } * * * * \quad 0 \quad * \quad * \quad *
\end{aligned}
$$

In this paper we call a system $\{\mathbf{a}, \mathbf{b}\}$ of arrays $\mathbf{a}, \mathbf{b} \in l\left(\boldsymbol{Z}_{N_{1}} \times \boldsymbol{Z}_{N_{2}}\right)$ an orientation selective system if their frequencies are localized in either $I_{1} \cup I_{3} \cup \Delta$ or $I_{2} \cup I_{4} \cup \Delta$, and $\mathcal{F}_{N_{1}, N_{2}}(\mathbf{a})+\mathcal{F}_{N_{1}, N_{2}}(\mathbf{b})=\mathbf{1}_{N_{1} \times N_{2}}$. In this paper we propose two types of orientation selective filters. One of these is defined by using the following arrays:

$$
\begin{gathered}
\widetilde{A}\left(n_{1}, n_{2}\right)= \begin{cases}1, & \left(n_{1}, n_{2}\right) \in I_{1} \cup I_{3} \\
0, & \left(n_{1}, n_{2}\right) \in I_{2} \cup I_{4} \\
\frac{1}{2}, & \left(n_{1}, n_{2}\right) \in \Delta\end{cases} \\
\widetilde{B}\left(n_{1}, n_{2}\right)= \begin{cases}0, & \left(n_{1}, n_{2}\right) \in I_{1} \cup I_{3} \\
1, & \left(n_{1}, n_{2}\right) \in I_{2} \cup I_{4} \\
\frac{1}{2}, & \left(n_{1}, n_{2}\right) \in \Delta\end{cases}
\end{gathered}
$$

Then let $\widetilde{\mathbf{a}}=\mathcal{F}_{N_{1}, N_{2}}^{-1}(\widetilde{A})$ and $\widetilde{\mathbf{b}}=\mathcal{F}_{N_{1}, N_{2}}^{-1}(\widetilde{\boldsymbol{B}})$. By this definition we have that $\{\widetilde{\mathbf{a}}, \widetilde{\mathbf{b}}\}$ is an orientation selective system. We call it the ideal orientation selective system. Frequencies of $\widetilde{\mathbf{a}}$ and $\widetilde{\mathbf{b}}$ are perfectly localized in $I_{1} \cup I_{3} \cup \Delta$ and $I_{2} \cup I_{4} \cup \Delta$ respectively. However by computation it is easy to see that non-zero elements of $\widetilde{\mathbf{a}}$ and $\tilde{\mathbf{b}}$ spread intermittently over the whole space $\boldsymbol{Z}_{N_{1}} \times \boldsymbol{Z}_{N_{2}}$. In this paper we propose an orientation selective system whose all nonzero elements are packed in a small subarea of $\boldsymbol{Z}_{N_{1}} \times \boldsymbol{Z}_{N_{2}}$. One of ideas is to approximate $\widetilde{A}$ or $\widetilde{B}$ by using discretized real analytic functions, and to take a partial sum of finite Fourier series of them. Let $s_{1}(x)=\sin (x)$ and

$$
s_{p}(x)=\frac{1}{\sin (1)} \sin \left(s_{p-1}(x)\right)
$$

for $p=2,3, \ldots$. The function $s_{p}(x)$ was studied by Gray and Glynn ([12]) from a complete different interest. They showed that when $x \neq \pi n, n \in \boldsymbol{Z}$, the sequence $\left\{s_{p}(x)\right\}_{p=1,2, \ldots}$ approximates to the $2 \pi$-periodic step function $\chi$ satisfying

$$
\chi(x)= \begin{cases}1, & x \in[0, \pi), \\ -1, & x \in[\pi, 2 \pi) .\end{cases}
$$

The desired orientation selective system is constructed by the following way. Let

$$
S_{p}^{l}\left[n_{l}\right]=s_{p}\left(2 \pi \frac{n_{l}}{N_{l}}\right), n_{l} \in Z_{N_{l}}, l=1,2,
$$

and $\delta_{p}^{l}=\left(S_{p}^{l}\left[n_{l}\right]\right)_{n_{l} \in \boldsymbol{Z}_{N_{l}}}$. Let apprx and $t h r$ be positive numbers. We use these numbers as parameters controlled the size and performance of orientation selective system, and their values will be determined later on. Let 

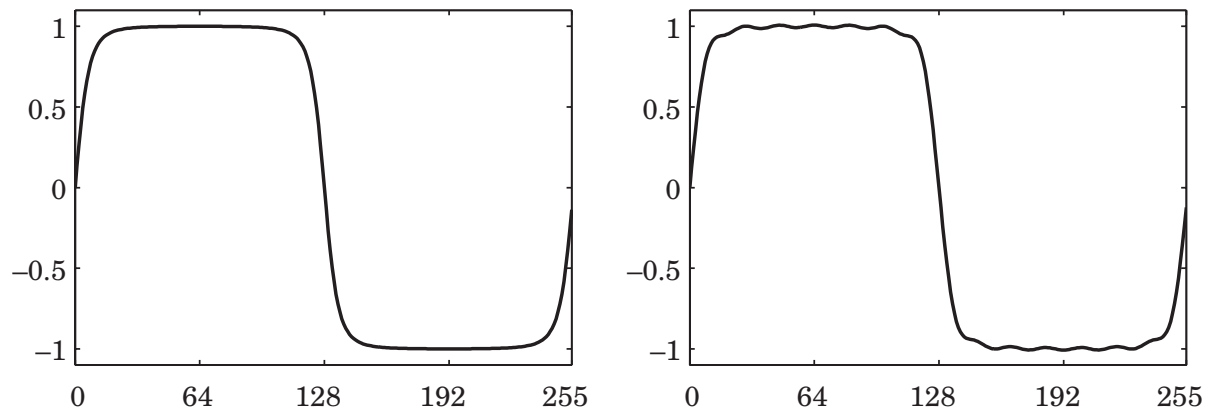

Fig. 5. Left: the graph of $f_{11}^{1}$. Right: the graph of $f_{11}^{1, \text { apprx }}$, apprx $=10 . N_{1}=256$.

$$
q_{l}=\min \left\{q: \sum_{k=q}^{N_{l} / 2}\left|\mathcal{F}_{N_{l}}\left(g_{p}^{l}\right)[k]\right|^{2}<\operatorname{apprx}\right\} .
$$

We assume that apprx is determined so that $0<q_{l}<N_{l} / 2$.

For $k \in Z_{N_{l}}$, let

$$
J_{q_{l}}[k]= \begin{cases}0, & q_{l} \leq k \leq N_{l}-q_{l}, \\ 1, & \text { otherwise, }\end{cases}
$$

and $\mathbf{J}_{q_{l}}=\left(J_{q_{l}}[k]\right)_{k \in Z_{N_{l}}}$. Then $\mathbf{J}_{q_{l}}$ is a lowpass filter. Define

$$
s_{p}^{l, a p p r x}=\mathcal{F}_{N_{l}}^{-1}\left(\mathcal{F}_{N_{l}}\left(s_{p}^{l}\right) . \mathbf{J}_{q_{l}}\right) .
$$

It is easy to see that $8_{p}^{l, a p p r x}$ is a partial sum of the finite Fourier series of $f_{p}^{l}$. As is well known, usual Fourier series of a discontinuous function such as the function $\chi$ raises the so-called Gibbs phenomenon. However, since $s_{p}(x)$ is a real analytic function, its Fourier series converges uniformly to $s_{p}(x)$, that is, the Gibbs phenomenon does not happen. Moreover magnitude of its high frequency part decays very fast. This is an advantage of using $S_{p}^{l, a p p r x}$ instead of adopting directly Fourier approximations of the characteristic function $\chi$.

In order to construct our orientation selective system we define the following filter:

$$
\mathbf{x a}_{p}^{l}=-\frac{1}{2} \rho_{p}^{l}+\frac{1}{2} \mathbf{1}_{N_{l}}
$$

In the previous section we constructed the subband decomposition $\left\{\boldsymbol{F}_{j}\left(\mu_{1}, \mu_{2}, \mathbf{x}\right): j=1, \ldots, J, \mu_{1}, \mu_{2}=0, \ldots, m\right\}$ for a $2 \mathrm{D}$ array $\mathbf{x} \in l\left(\boldsymbol{Z}_{N_{1}} \times \boldsymbol{Z}_{N_{1}}\right)$. By analogous way as this $2 \mathrm{D}$ case, for $1 \mathrm{D}$ array $\mathbf{x} \in l\left(\boldsymbol{Z}_{N_{l}}\right)(l=1,2)$ we can define $1 \mathrm{D}$ version of the subband decomposition. We denote it by $\left\{\boldsymbol{F}_{j}^{(l)}(\mu, \mathbf{x}), j=1, \ldots, J, \mu=0, \ldots, m\right\}, l=1,2$. Let $\delta \in l\left(\boldsymbol{Z}_{N_{l}}\right)$ be the unit impulse, and for each $\mu$, let

$$
p_{c}\left(=p_{c}(\mu, j, l)\right)=\min \left\{p \geq 2: \max _{k=1, \ldots, N_{l} / 2-1}\left|\tau_{N_{l} / 2}^{l}\left(\mathcal{F}_{N_{l}}\left(\mathbf{F}_{j}^{(l)}(\mu, \delta)\right)\right)[k] \mathbf{x a}_{p}^{l}[k]\right|<t h r\right\},
$$

where $\min \varnothing=+\infty$. In this paper we assume that $p_{c}$ is finite. In fact, in our computer experiments described later, we take $t h r$ so that $p_{c}<\infty$. Now for $l=1,2, j=1, \ldots, J$ and $\mu=0, \ldots, m$, let

$$
\alpha_{\mu}^{l, j}=\frac{i}{\sqrt{2}} \oiint_{p_{c}}^{l, a p p r x}
$$

For $j=1, \ldots, J$, and $\mu_{1}, \mu_{2}=2, \ldots, m$, let

$$
\begin{aligned}
& \mathcal{A}_{\mu_{1}, \mu_{2}}^{j}\left(=\mathcal{A}_{\mu_{1}, \mu_{2}}^{j}(\text { apprx }, \text { thr })\right)=\alpha_{\mu_{1}}^{1, j} \otimes \alpha_{\mu_{2}}^{2, j}+\frac{1}{2} \mathbf{1}_{N_{1}, N_{2}}, \\
& \mathcal{B}_{\mu_{1}, \mu_{2}}^{j}\left(=\mathcal{B}_{\mu_{1}, \mu_{2}}^{j}(\text { apprx }, \text { thr })\right)=-\alpha_{\mu_{1}}^{1, j} \otimes \alpha_{\mu_{2}}^{2, j}+\frac{1}{2} \mathbf{1}_{N_{1}, N_{2}} .
\end{aligned}
$$

Let

$$
\mathbf{a}_{\mu_{1}, \mu_{2}}^{j}=\mathcal{F}_{N_{1}, N_{2}}^{-1}\left(\mathcal{A}_{\mu_{1}, \mu_{2}}^{j}\right) \text {, and } \mathbf{b}_{\mu_{1}, \mu_{2}}^{j}=\mathcal{F}_{N_{1}, N_{2}}^{-1}\left(\mathcal{B}_{\mu_{1}, \mu_{2}}^{j}\right) \text {. }
$$

We call the family of filters $\left\{\mathbf{a}_{\mu_{1}, \mu_{2}}^{j}, \mathbf{b}_{\mu_{1}, \mu_{2}}^{j}\right\}_{j, \mu_{1}, \mu_{2}}$ an adaptive orientation selective system.

Now we can construct a 2D directional filterbank. Firstly we define the analysis bank. We decompose $\mathbf{F}_{j}\left(\mu_{1}, \mu_{2}\right)$ for $\mu_{1} \mu_{2}>0$ by using the orientation selective system $\left\{\mathbf{a}_{\mu_{1}, \mu_{2}}^{j}, \mathbf{b}_{\mu_{1}, \mu_{2}}^{j}\right\}$ we have constructed as follows: 


$$
\begin{aligned}
& \mathbf{F}_{j}\left(\mu_{1}, \mu_{2}, a, \mathbf{x}\right)=\mathcal{F}_{N_{1}, N_{2}}^{-1}\left(\tau^{-1}\left(\mathcal{F}_{N_{1}, N_{2}}\left(\mathbf{a}_{\mu_{1}, \mu_{2}}^{j}\right) \tau \tau\left(\mathcal{F}_{N_{1}, N_{2}}\left(\mathbf{F}_{j}\left(\mu_{1}, \mu_{2}\right)\right)\right)\right)\right), \\
& \mathbf{F}_{j}\left(\mu_{1}, \mu_{2}, b, \mathbf{x}\right)=\mathcal{F}_{N_{1}, N_{2}}^{-1}\left(\tau^{-1}\left(\mathcal{F}_{N_{1}, N_{2}}\left(\mathbf{b}_{\mu_{1}, \mu_{2}}^{j}\right) \tau\left(\mathcal{F}_{N_{1}, N_{2}}\left(\mathbf{F}_{j}\left(\mu_{1}, \mu_{2}\right)\right)\right)\right) .\right.
\end{aligned}
$$

We abbreviate $\mathbf{F}_{j}\left(\mu_{1}, \mu_{2}, \varepsilon, \mathbf{x}\right)$ to $\mathbf{F}_{j}\left(\mu_{1}, \mu_{2}, \varepsilon\right)$. It is easy to see that

$$
\begin{aligned}
& \mathbf{F}_{j}\left(\mu_{1}, \mu_{2}, a\right)=\mathcal{F}_{N_{1}, N_{2}}^{-1}\left(\tau^{-1}\left(\mathcal{F}_{N_{1}, N_{2}}\left(\mathbf{a}_{\mu_{1}, \mu_{2}}^{j}\right)\right) \mathcal{F}_{N_{1}, N_{2}}\left(\mathbf{F}_{j}\left(\mu_{1}, \mu_{2}\right)\right)\right), \\
& \mathbf{F}_{j}\left(\mu_{1}, \mu_{2}, b\right)=\mathcal{F}_{N_{1}, N_{2}}^{-1}\left(\tau^{-1}\left(\mathcal{F}_{N_{1}, N_{2}}\left(\mathbf{b}_{\mu_{1}, \mu_{2}}^{j}\right)\right) \mathcal{F}_{N_{1}, N_{2}}\left(\mathbf{F}_{j}\left(\mu_{1}, \mu_{2}\right)\right)\right) .
\end{aligned}
$$

Therefore the mapping defined by $\mathbf{x} \mapsto \mathbf{F}_{j}\left(\mu_{1}, \mu_{2}, \varepsilon\right)$ is linear and shift-invariant. We depict in Section 6 the unit impulse response of $\mathbf{F}_{j}\left(\mu_{1}, \mu_{2}, \varepsilon\right)$ defined by using the filters in Example 1 (see Fig. 7). Further, in Section 7 we show how $\mathbf{F}_{j}\left(\mu_{1}, \mu_{2}, \varepsilon\right)$ 's extract components in orientation from a given image.

Next we construct the synthesis bank. Let $\widetilde{g}^{(\mu)}=\left(\widetilde{g}_{l}^{(\mu)}[n]\right)_{n \in \boldsymbol{Z}}=g^{(\mu)}, \mu=0,1, \ldots, m$. For $\varepsilon=a, b$, let

$$
\begin{aligned}
\widetilde{\mathbf{F}}_{J}\left(\mu_{1}, \mu_{2}, \varepsilon\right) & =\frac{1}{4^{J}} \widetilde{f}_{1}^{0,0}\left(\cdots \widetilde{f}_{J-1}^{0,0}\left(\widetilde{f}_{J}^{\mu_{1}, \mu_{2}}\left(\mathbf{F}_{J}\left(\mu_{1}, \mu_{2}, \varepsilon\right)\right)\right) \cdots\right), \\
\widetilde{\mathbf{F}}_{J-1}\left(\mu_{1}, \mu_{2}, \varepsilon\right) & =\frac{1}{4^{J-1}} \widetilde{f}_{1}^{0,0}\left(\cdots \widetilde{f}_{J-2}^{0,0}\left(\widetilde{f}_{J-1}^{\mu_{1}, \mu_{2}}\left(\mathbf{F}_{J-1}\left(\mu_{1}, \mu_{2}, \varepsilon\right)\right)\right) \cdots\right), \\
& \vdots \\
\widetilde{\mathbf{F}}_{1}\left(\mu_{1}, \mu_{2}, \varepsilon\right) & =\frac{1}{4} \widetilde{f}_{1}^{\mu_{1}, \mu_{2}}\left(\mathbf{F}_{1}\left(\mu_{1}, \mu_{2}, \varepsilon\right)\right) .
\end{aligned}
$$

Since

$$
\mathbf{F}_{j}\left(\mu_{1}, \mu_{2}\right)=\mathbf{F}_{j}\left(\mu_{1}, \mu_{2}, a\right)+\mathbf{F}_{j}\left(\mu_{1}, \mu_{2}, b\right),
$$

we have that

$$
\mathbf{x}=\widetilde{\mathbf{F}}_{J}(0,0)+\sum_{j=1}^{J} \sum_{\substack{\mu_{1}, \mu_{2}=0, \mu_{1} \mu_{2}=0 \\ \mu_{1}+\mu_{2} \neq 0}}^{m} \widetilde{\mathbf{F}}_{j}\left(\mu_{1}, \mu_{2}\right)+\sum_{j=1}^{J} \sum_{\substack{\mu_{1}, \mu_{2}=0 \\ \mu_{1} \mu_{2}>0}}^{m}\left(\widetilde{\mathbf{F}}_{j}\left(\mu_{1}, \mu_{2}, a\right)+\widetilde{\mathbf{F}}_{j}\left(\mu_{1}, \mu_{2}, b\right)\right) .
$$

We call (10) the orientation selective multi-resolution decomposition.

Note that although our procedure can be applied to filters $g^{(\mu)}$ and $\widetilde{g}^{(\mu)}, \mu=0,1, \ldots, m$, satisfying the condition (2), we think by the nature of our construction the filterbank is possible to have good orientation selectivity, when $g^{(0)}$ is a lowpass filter and $g^{(1)}, \ldots, g^{(m)}$ are bandpass filters such that the passband of $g^{(j)}$ is in a higher frequency area than one of $g^{(k)}$ when $k<j$. For instance we can construct a 2D filterbank with orientation selectivity by using filters in Example 1. There are other choices: for example we can adopt also framelet filters proposed in Selesnick and Abdelnour [28].

\section{A Realization of Shift-Invariant Directional Filterbanks by Tensor Product}

As we have seen in Section 3, subband signals $\mathbf{F}_{j}\left(\mu_{1}, \mu_{2}\right)$ are produced from linear combinations of tensor products of $1 \mathrm{D}$ filters. In this section we show that each subband signals $\mathbf{F}_{j}\left(\mu_{1}, \mu_{2}, \varepsilon\right)$ for the orientation selective system $\left\{\mathbf{a}_{\mu_{1}, \mu_{2}}^{j}, \mathbf{b}_{\mu_{1}, \mu_{2}}^{j}\right\}$ are obtained also by linear combinations of tensor products of 1D filters. For $l=1,2, j=1, \ldots, J$ and $\mu=0, \ldots, m$, let $\mathbf{a}_{\mu}^{l, j}=\mathcal{F}_{N_{l}}^{-1}\left(\alpha_{\mu}^{l, j}\right)$. Then it is easy to see that $\mathbf{a}_{\mu}^{l, j}$ is a real valued FIR filter and its length is $2 q_{l}-1$ (see (9) for the definition of $q_{l}$ ). Since

$$
\begin{aligned}
& \mathcal{A}_{\mu_{1}, \mu_{2}}^{j}=\alpha_{\mu_{1}}^{1, j} \otimes \alpha_{\mu_{2}}^{2, j}+\frac{1}{2} \mathbf{1}_{N_{1}, N_{2}}, \\
& \mathcal{B}_{\mu_{1}, \mu_{2}}^{j}=-\alpha_{\mu_{1}}^{1, j} \otimes \alpha_{\mu_{2}}^{2, j}+\frac{1}{2} \mathbf{1}_{N_{1}, N_{2}}, \\
& \mathbf{a}_{\mu_{1}, \mu_{2}}^{j}=\mathcal{F}_{N_{1}, N_{2}}^{-1}\left(\mathcal{A}_{\mu_{1}, \mu_{2}}^{j}\right), \text { and } \mathbf{b}_{\mu_{1}, \mu_{2}}^{j}=\mathcal{F}_{N_{1}, N_{2}}^{-1}\left(\mathcal{B}_{\mu_{1}, \mu_{2}}^{j}\right),
\end{aligned}
$$

we have that

$$
\begin{aligned}
\mathbf{a}_{\mu_{1}, \mu_{2}}^{j} & =\mathcal{F}_{N_{1}}^{-1}\left(\alpha_{\mu_{1}}^{1, j}\right) \otimes \mathcal{F}_{N_{2}}^{-1}\left(\alpha_{\mu_{2}}^{2, j}\right)+\frac{1}{2} \delta^{1} \otimes \delta^{2} \\
& =\mathbf{a}_{\mu_{1}}^{1, j} \otimes \mathbf{a}_{\mu_{2}}^{2, j}+\frac{1}{2} \delta^{1} \otimes \delta^{2}
\end{aligned}
$$

where $\delta^{l}$ is the unit impulse, that is $\delta^{l}=(1,0, \ldots, 0) \in l\left(\boldsymbol{Z}_{N_{l}}\right), l=1,2$.

Since it is easy to see that $\tau^{-1}\left(\mathcal{F}_{N_{1}, N_{2}}\left(\mathbf{a}_{\mu_{1}, \mu_{2}}^{j}\right)\right)=\mathcal{F}_{N_{1}, N_{2}}\left(\mathbf{a}_{\mu_{1}, \mu_{2}}^{j}\right)$, we have 


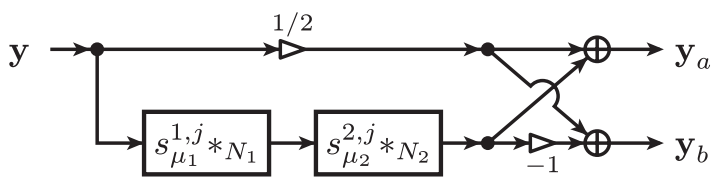

Fig. 6.

$$
\begin{aligned}
\mathbf{F}_{j}\left(\mu_{1}, \mu_{2}, a\right) & =\mathcal{F}_{N_{1}, N_{2}}^{-1}\left(\tau^{-1}\left(\mathcal{F}_{N_{1}, N_{2}}\left(\mathbf{a}_{\mu_{1}, \mu_{2}}^{j}\right)\right)_{\bullet} \mathcal{F}_{N_{1}, N_{2}}\left(\mathbf{F}_{j}\left(\mu_{1}, \mu_{2}\right)\right)\right) \\
& =\mathcal{F}_{N_{1}, N_{2}}^{-1}\left(\mathcal{F}_{N_{1}, N_{2}}\left(\mathbf{a}_{\mu_{1}, \mu_{2}}^{j}\right) . \mathcal{F}_{N_{1}, N_{2}}\left(\mathbf{F}_{j}\left(\mu_{1}, \mu_{2}\right)\right)\right) \\
& =\mathbf{a}_{\mu_{1}, \mu_{2}}^{j} *_{N_{1}, N_{2}} \mathbf{F}_{j}\left(\mu_{1}, \mu_{2}\right) \\
& =\mathbf{a}_{\mu_{1}}^{1, j} \otimes \mathbf{a}_{\mu_{2}}^{2, j} *_{N_{1}, N_{2}} \mathbf{F}_{j}\left(\mu_{1}, \mu_{2}\right)+\frac{1}{2} \mathbf{F}_{j}\left(\mu_{1}, \mu_{2}\right),
\end{aligned}
$$

and

$$
\mathbf{F}_{j}\left(\mu_{1}, \mu_{2}, b\right)=-\mathbf{a}_{\mu_{1}}^{1, j} \otimes \mathbf{a}_{\mu_{2}}^{2, j} *_{N_{1}, N_{2}} \mathbf{F}_{j}\left(\mu_{1}, \mu_{2}\right)+\frac{1}{2} \mathbf{F}_{j}\left(\mu_{1}, \mu_{2}\right)
$$

Now, for a signal $\mathbf{y}$ in $l\left(\boldsymbol{Z}_{N_{1}} \times \boldsymbol{Z}_{N_{2}}\right)$, let $\mathbf{y}_{a}$ and $\mathbf{y}_{b}$ be defined by the diagram indicated in Figure 6 .

Write $\mathbf{y}_{b}=S_{\mu_{1}, \mu_{2}}^{j, b}(\mathbf{y})$ and $\mathbf{y}_{a}=S_{\mu_{1}, \mu_{2}}^{j, a}(\mathbf{y})$. Then what we have seen implies the following proposition.

\section{Proposition 3.}

$$
\begin{aligned}
& \mathbf{F}_{j}\left(\mu_{1}, \mu_{2}, a\right)=S_{\mu_{1}, \mu_{2}}^{j, a}\left(\mathbf{F}_{j}\left(\mu_{1}, \mu_{2}\right)\right), \\
& \mathbf{F}_{j}\left(\mu_{1}, \mu_{2}, b\right)=S_{\mu_{1}, \mu_{2}}^{j, b}\left(\mathbf{F}_{j}\left(\mu_{1}, \mu_{2}\right)\right) .
\end{aligned}
$$

\section{Figures of 2D Filters}

In this section we show how filters produced by our method are tuned in orientation and spatial-frequency. For this purpose we depict impulse responses of approximation and detail coefficients for an FPRMO filterbank. As an example, we here consider $\left\{g_{m}^{(0)}, \ldots, g_{m}^{(m)} ; g_{m}^{(0)}, \ldots, g_{m}^{(m)}\right\}$ in Example 1 with $m=6$, and we take apprx $=10$, and $t h r=0.075$ (for their definitions, see Section 4). By our computer experiments, these values of apprx and thr achieve satisfactory orientation selectivity. Let $\boldsymbol{\Delta}=\left(\Delta\left[n_{1}, n_{2}\right]\right)_{n_{1} \in \boldsymbol{Z}_{256}, n_{2} \in \boldsymbol{Z}_{256}}$ be the $2 \mathrm{D}$ unit impulse defined by

$$
\boldsymbol{\Delta}\left(n_{1}, n_{2}\right)= \begin{cases}1, & n_{1}=n_{2}=129, \\ 0, & \text { otherwise. }\end{cases}
$$

Graphs of impulse responses $\mathbf{F}_{2}\left(\mu_{1}, \mu_{2}, \boldsymbol{\Delta}\right), \mu_{1} \mu_{2}=0, \mu_{1}, \mu_{2}=0, \ldots, 6$, and $\mathbf{F}_{2}\left(\mu_{1}, \mu_{2}, \varepsilon, \boldsymbol{\Delta}\right), \mu_{1} \mu_{2} \geq 1, \mu_{1}, \mu_{2}=$ $0, \ldots, 6$ are shown in Fig. 7. In Fig. 8 we can see their spectra. As is indicated by Fig. 7, impulse responses are tuned to almost all directions.

The above observation is of the case of $m=6$. Now, what happens in other cases? When we choose as $m$ a bigger number, we can observe in fact that the higher efficiency of orientation selectivity is achieved. This phenomenon is attributed to that if $\mu$ tends from 0 to $m$, the spectra of $\mathbf{F}_{j}(0, \mu, \boldsymbol{\Delta})$ and $\mathbf{F}_{j}(\mu, 0, \boldsymbol{\Delta})$ ascend more gradually but finely than the case of $m$ being smaller.

In the next section we check actual performance of orientation selectivity of the filterbank by processing a test image. And in Section 8 we discuss the filterbanks from the viewpoint of vision science.

\section{Discussion from the Viewpoint of Image Processing}

In this section we show how our filterbank decomposes an image into components in orientation. As in the previous section, let $\left\{g_{6}^{(0)}, \ldots, g_{6}^{(6)} ; g_{6}^{(0)}, \ldots, g_{6}^{(6)}\right\}$ be filters described in Example 1, and let apprx $=10$, and $t h r=0.075$. Here we deal with the image $\mathbf{x}$, of size $256 \times 256$ pixels, shown in Fig. 9. It contains three ties on which stripes in various orientations are designed.

We apply our filterbank to the image $\mathbf{x}$. Four of resulting subband signals are shown in Fig. 10: $\mathbf{F}_{2}(5,2, a, \mathbf{x})($ top of the left), $\mathbf{F}_{2}(2,5, a, \mathbf{x})$ (bottom of the left), $\mathbf{F}_{2}(5,2, b, \mathbf{x})$ (top of the right), and $\mathbf{F}_{2}(2,5, b, \mathbf{x})$ (bottom of the right). Graphs of corresponding impulse responses used for getting these subband signals are illustrated in Fig. 11.

By Fig. 10 we can recognize stripes to the middle tie in Fig. 9 are extracted mainly by $\mathbf{F}_{2}(5,2, b, \mathbf{x})$. However we hardly can find these stripes on $\mathbf{F}_{2}(5,2, a, \mathbf{x})$. Moreover, crossed stripes of the tie in the left in Fig. 9 are separated into $\mathbf{F}_{2}(5,2, a, \mathbf{x})$ and $\mathbf{F}_{2}(2,5, b, \mathbf{x})$, edges of the tie on the left are extracted by $\mathbf{F}_{2}(2,5, b, \mathbf{x})$, and edges of the tie in the 


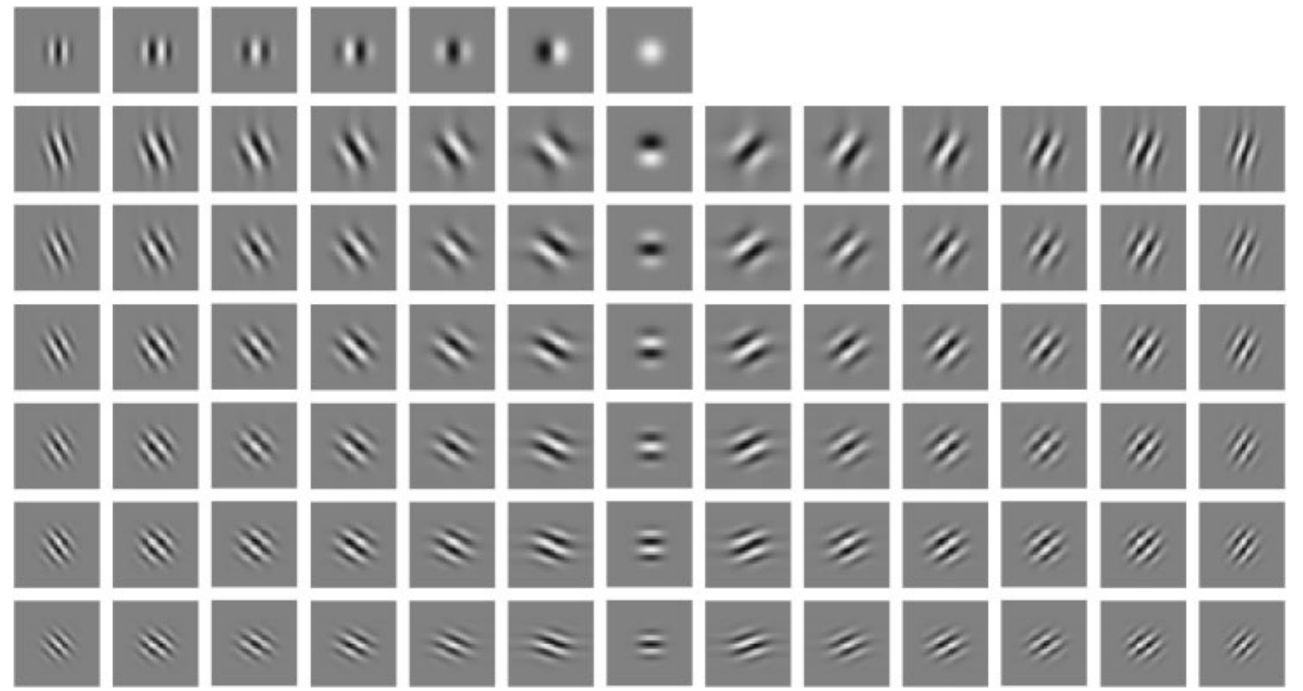

Fig. 7. Graphs of $\mathbf{F}_{2}\left(\mu_{1}, \mu_{2}, \boldsymbol{\Delta}\right), \mu_{1} \mu_{2}=0$, and $\mathbf{F}_{2}\left(\mu_{1}, \mu_{2}, \varepsilon, \boldsymbol{\Delta}\right), \mu_{1} \mu_{2} \geq 1$. Each figure $(32 \times 32$ pixels $)$ is the central portion of $256 \times 256$ image.

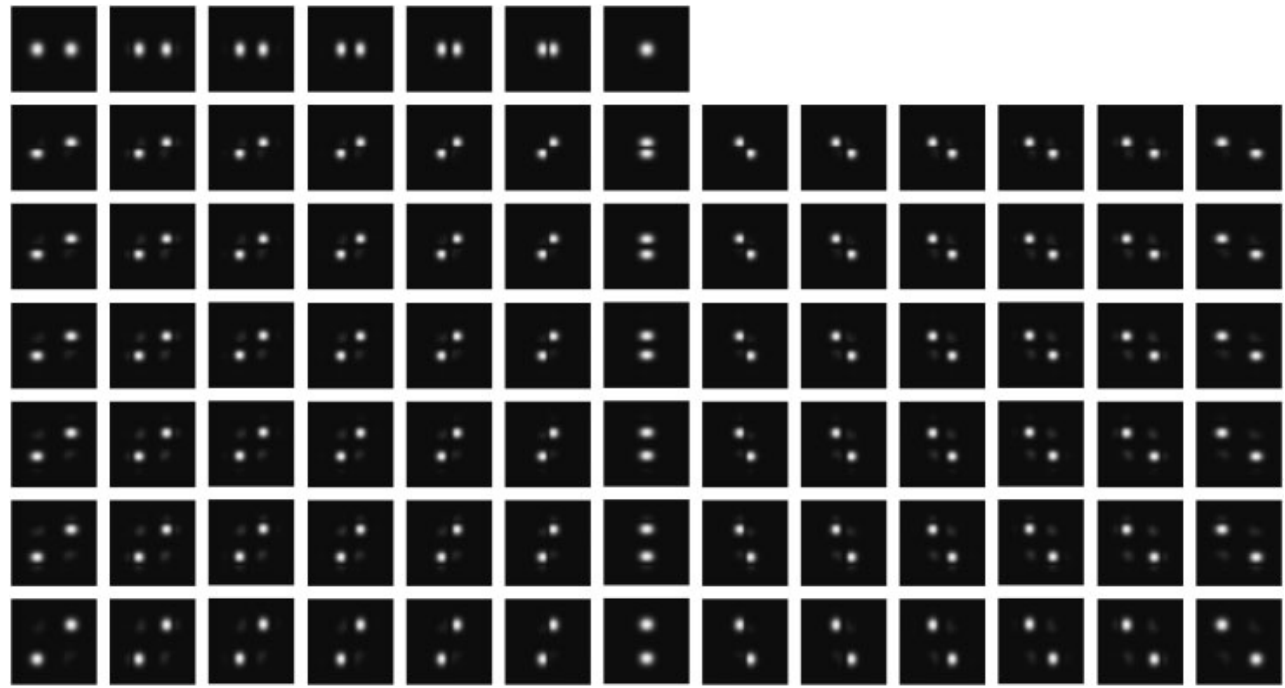

Fig. 8. Magnitude of DFT spectra of $\mathbf{F}_{2}\left(\mu_{1}, \mu_{2}, \boldsymbol{\Delta}\right), \mu_{1} \mu_{2}=0$, and $\mathbf{F}_{2}\left(\mu_{1}, \mu_{2}, \varepsilon, \boldsymbol{\Delta}\right), \mu_{1} \mu_{2} \geq 1$.

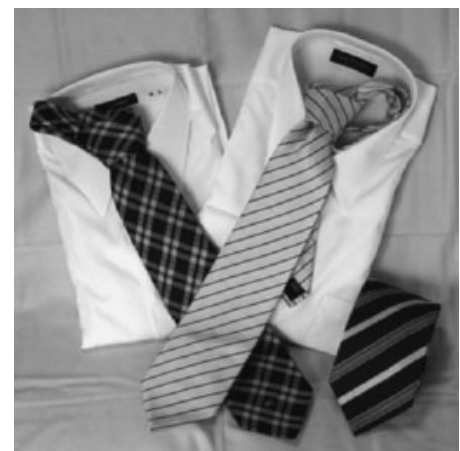

Fig. 9.

middle by $\mathbf{F}_{2}(5,2, b, \mathbf{x})$. These show that the filterbank has high ability of selecting orientation.

In this computer experiment we have set apprx $=10$ and $t h r=0.075$, but what happens in the case of other values? To begin from the conclusion, orientation selectivity is improved if apprx and thr are smaller. The reason is that if apprx and thr decrease, then the adaptive orientation selective system is more and more close to the ideal one. The ideal one achieves the highest performance of selecting orientation, because by the definition it separates the frequency 

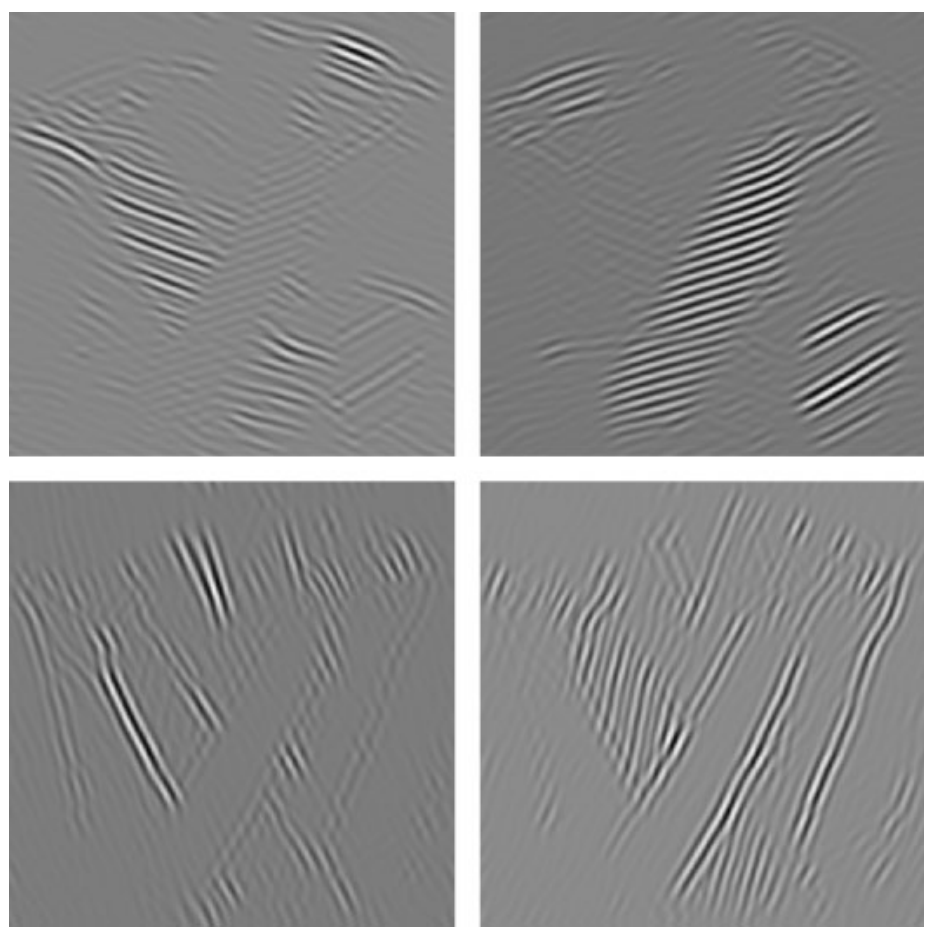

Fig. 10. Top of the left: $\mathbf{F}_{2}(5,2, a, \mathbf{x})$, bottom of the left: $\mathbf{F}_{2}(2,5, a, \mathbf{x})$, top of the right: $\mathbf{F}_{2}(5,2, b, \mathbf{x})$, bottom of the right $\mathbf{F}_{2}(2,5, b, \mathbf{x})$.
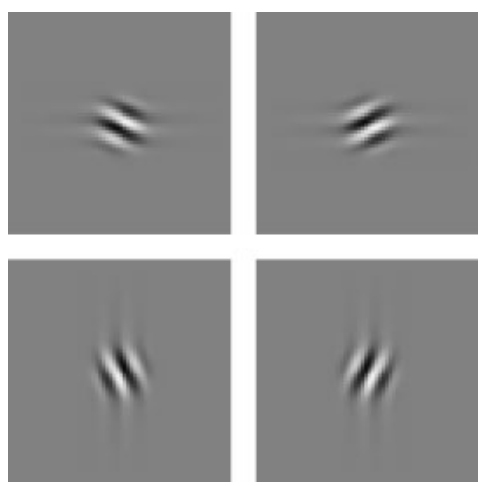

Fig. 11. Impulse responses of $2 \mathrm{D}$ filters: top of the left is $\mathbf{F}_{2}(5,2, a, \mathbf{\Delta})$, bottom of the left $\mathbf{F}_{2}(2,5, a, \boldsymbol{\Delta})$, top of the right $\mathbf{F}_{2}(5,2, b, \boldsymbol{\Delta})$, and bottom of the right $\mathbf{F}_{2}(2,5, b, \boldsymbol{\Delta})$. Each is the central portion of size $64 \times 64$ of the image of size $256 \times 256$.

domain perfectly into two disparate opposite regions. We compare performance of two systems, adaptive one and ideal one. For this purpose, let us concentrate our attention to inclined stripes of the tie in the middle in Fig. 9. As we have seen in Fig. 10, in the former case, these stripes are extracted almost by $\mathbf{F}_{2}(5,2, b, \boldsymbol{\Delta})$. However this separation is not perfect: by careful observation, we see these stripes are also picked up a bit by $\mathbf{F}_{2}(5,2, a, \boldsymbol{\Delta})$, although the orientation preference of $\mathbf{F}_{2}(5,2, b, \boldsymbol{\Delta})$ is opposite to one of $\mathbf{F}_{2}(5,2, a, \boldsymbol{\Delta})$. By contraries, in the latter case, we can not find these stripes in $\mathbf{F}_{2}(5,2, a, \mathbf{x})$ shown in Fig. 12. There are other evidences which indicate the superior performance of the ideal orientation selective system: edges of ties constitute inclined lines, and for example, the ideal one extracts edges of the tie in the middle perfectly by $\mathbf{F}_{2}(2,5, b, \mathbf{x})$ (see Fig. 12), while the adaptive one picks up these edges certainly by $\mathbf{F}_{2}(2,5, b, \mathbf{x})$, but they remain also faintly in the opposite $\mathbf{F}_{2}(2,5, a, \mathbf{x})$ (see Fig. 10).

Thus the orientation selectivity of ideal one is better than of adaptive one. Nevertheless an adaptive orientation selective system has an attractive advantage: it endows the filterbank with orientation selectivity, and at the same time can prevent sizes of filters of the filterbank from large dilatation. In fact, if one chooses small values as approx and thr, the orientation selectivity becomes better, while as its compensation, the sizes of filters in the adaptive orientation selective system become larger. The extreme case is the ideal orientation selective system. The sizes of filters in it are always $N_{1} \times N_{2}$ which is same as one of input images. See Fig. 13: the right is a perspective plot of $\mathbf{F}_{2}(5,2, a, \boldsymbol{\Delta})$ for the ideal one and the left for an adaptive one. The side lobe of the former spreads widely, while the latter is compactly supported. 

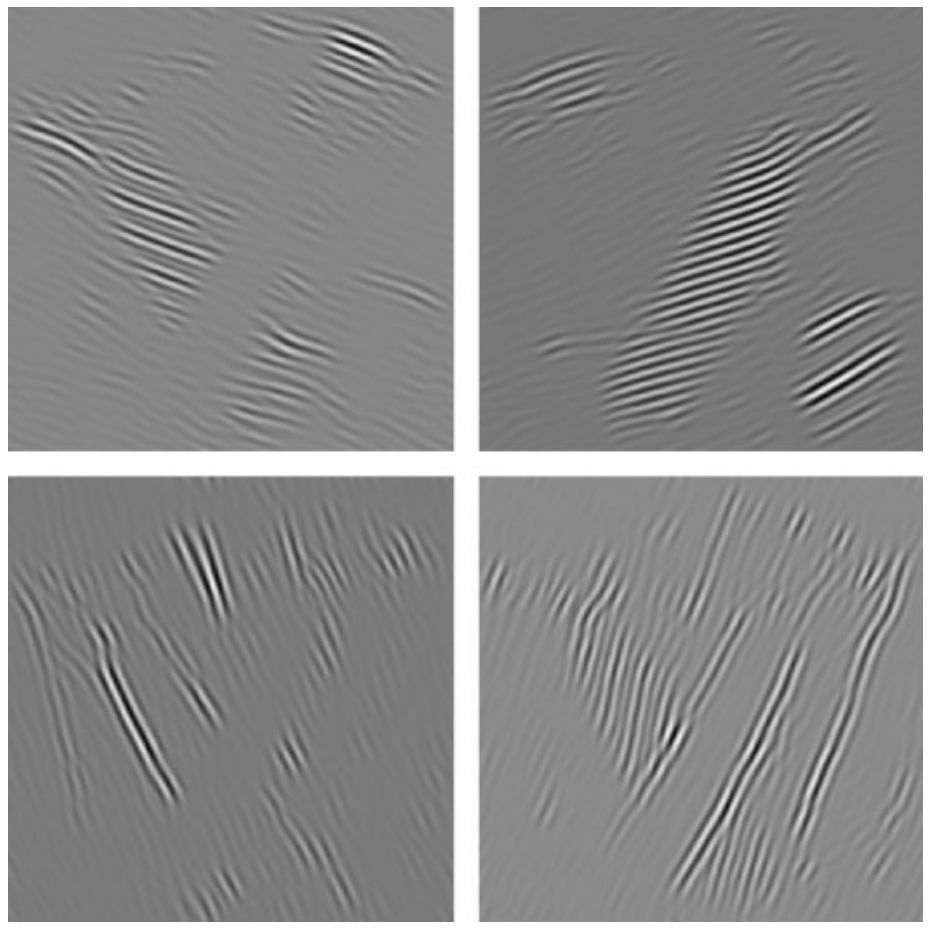

Fig. 12. The case of the ideal selective system. Top of the left: $\mathbf{F}_{2}(5,2, a, \mathbf{x})$, bottom of the left: $\mathbf{F}_{2}(2,5, a, \mathbf{x})$, top of the right: $\mathbf{F}_{2}(5,2, b, \mathbf{x})$, bottom of the right $\mathbf{F}_{2}(2,5, b, \mathbf{x})$.
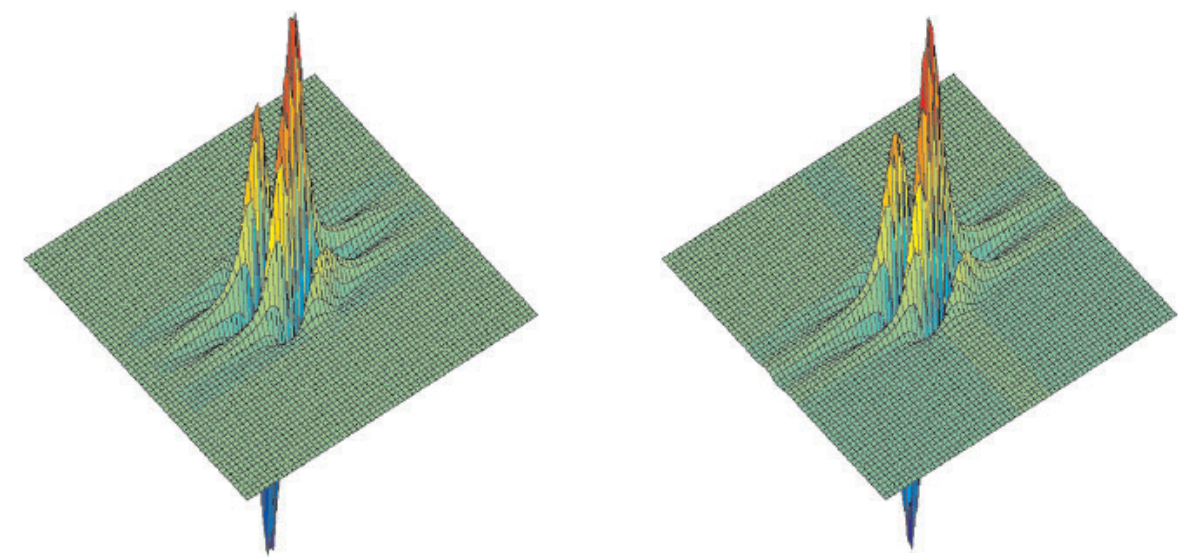

Fig. 13. Left: $\mathbf{F}_{2}(5,2, a, \mathbf{\Delta})$ (adaptive). Right: $\mathbf{F}_{2}(5,2, a, \mathbf{\Delta})$ (ideal). Each figure is the central portion of size $64 \times 64$ of the image of size $256 \times 256$ pixels.

\section{Discussion from the Viewpoint of Vision Science}

In this section we discuss our filterbanks from the viewpoint of vision science.

\section{Gaussian derivatives and their FIR analogues}

In Section 3-5, we proved that FPRMO filterbanks can be constructed from general 1D filters satisfying the condition (2). Firstly we discuss which 1D filters are appropriate to study of the vision. It is now generally accepted that profiles of receptive fields of simple cells in V1 are similar to the graphs of Gabor functions or derivatives of Gaussian functions ([30], [31]). However there is a mathematical problem with these functions. It is that they are supported by no bounded domains, that is, the area on which values of these functions are not zero spreads out over the infinite space. Since the extents of the receptive fields of simple cells are small, it is natural to model them rather by compactly supported functions. In addition, from the viewpoint of practical computation by computer, in order to use Gaussian derivatives and Gabor functions, we need to make discrete approximations of them, but this approximations collapse the perfect reconstruction property of the filterbank. As we will show later, the perfect reconstruction property is 
important for mathematical study of nonlinear processing in the brain. Thus it is desirable to adopt FIR filters which are like Gaussian derivatives. Here we see that 1D filters described in Example 1 are good candidates of such FIR filters: for example we are concerned with $g_{6}^{(0)}, \ldots, g_{6}^{(6)}$. Recall their construction ([26]). Let $B_{1}(x)$ be the characteristic function of the closed interval $\left[-\frac{1}{2}, \frac{1}{2}\right]$, and let

$$
B_{n}(x)=\int_{-\infty}^{\infty} B_{n-1}(x-y) B_{1}(y) d y, n=2,3, \ldots
$$

These functions are known as B-splines. For an integrable function $f$ on $\boldsymbol{R}$, its Fourier transform is defined by

$$
\widehat{f}(\xi)=\int_{-\infty}^{\infty} f(x) e^{-2 \pi i x \xi} d x, \xi \in \boldsymbol{R} .
$$

Let $\psi_{6}^{(\mu)}$ be the integrable function on $\boldsymbol{R}$ such that

$$
\widehat{\psi_{6}^{(\mu)}}(2 \xi)=G_{6}^{(\mu)}(\xi) \widehat{B_{6}}(\xi),
$$

where $G_{6}^{(\mu)}$ is the frequency response of $g_{6}^{(\mu)}, \mu=0, \ldots, 6$. Then by the spirit of Mallat's fast algorithm, $g_{6}^{(\mu)}$ is regarded as a discrete version of the function $\psi_{6}^{(\mu)}, \mu=0, \ldots, 6$. Graphs of $\psi_{6}^{(\mu)}, \mu=0, \ldots, 6$, are shown in Fig. 14. By comparing Fig. 14 with Fig. 15 which show graphs of Gaussian derivatives, one can recognize easily that the shape of the graph of each function $\psi_{6}^{(\mu)}$ is very similar to the $\mu$-th derivative of Gaussian function, $\mu=0, \ldots, 6$. We can also observe same similarity for $g_{m}^{(\mu)}$ with other $m$. From these reasons, we think that the 2D filterbanks constructed by using $g_{m}^{(\mu)}, \mu=0, \ldots, m$, are appropriate for the bases on which mathematical models of visual information processing are designed. The number $m$ should be determined by taking physiological facts into account. We will discuss in the next paragraph the number $m$. For convenience, in this paper we call $g_{m}^{(\mu)}$ the Gaussian-derivative-like filters of order $\mu$.

\section{The roles of FIR analogues of Gaussian derivatives}

So far, while roles of 1 st, 2nd and 3rd Gaussian derivatives have been extensively studied (cf. [11]), the role of higher derivatives is largely unknown. In general, what roles do filters $g_{m}^{(0)}, \ldots, g_{m}^{(m)}$ in our filterbank perform? We begin with addressing this issue from a pure mathematical viewpoint. It is easy to prove that these $m+1$ filters form a tight frame for $l\left(\boldsymbol{Z}_{N}\right)$ in the following sense: arrays $\mathbf{g}_{m, s}^{(\mu)}=\left(p_{N}\left(g_{m}^{(\mu)}\right)[k-s]\right)_{k \in \boldsymbol{Z}_{N}}, \mu=0, \ldots, m, s=0, \ldots, N-1$ are said to be a tight frame if there exists a positive constant $C$ such that

$$
\sum_{\mu=0}^{m} \sum_{s=0}^{N-1}\left|\left\langle\mathbf{g}_{m, s}^{(\mu)}, \mathbf{x}\right\rangle\right|^{2}=C \sum_{n=0}^{N-1}|x[n]|^{2}
$$

for every $\mathbf{x}=(x[n])_{n \in \boldsymbol{Z}_{N}}$, where $\langle\cdot, \cdot\rangle$ is the usual Hermitian inner product of $l\left(\boldsymbol{Z}_{N}\right)$. This means that the so-called frame
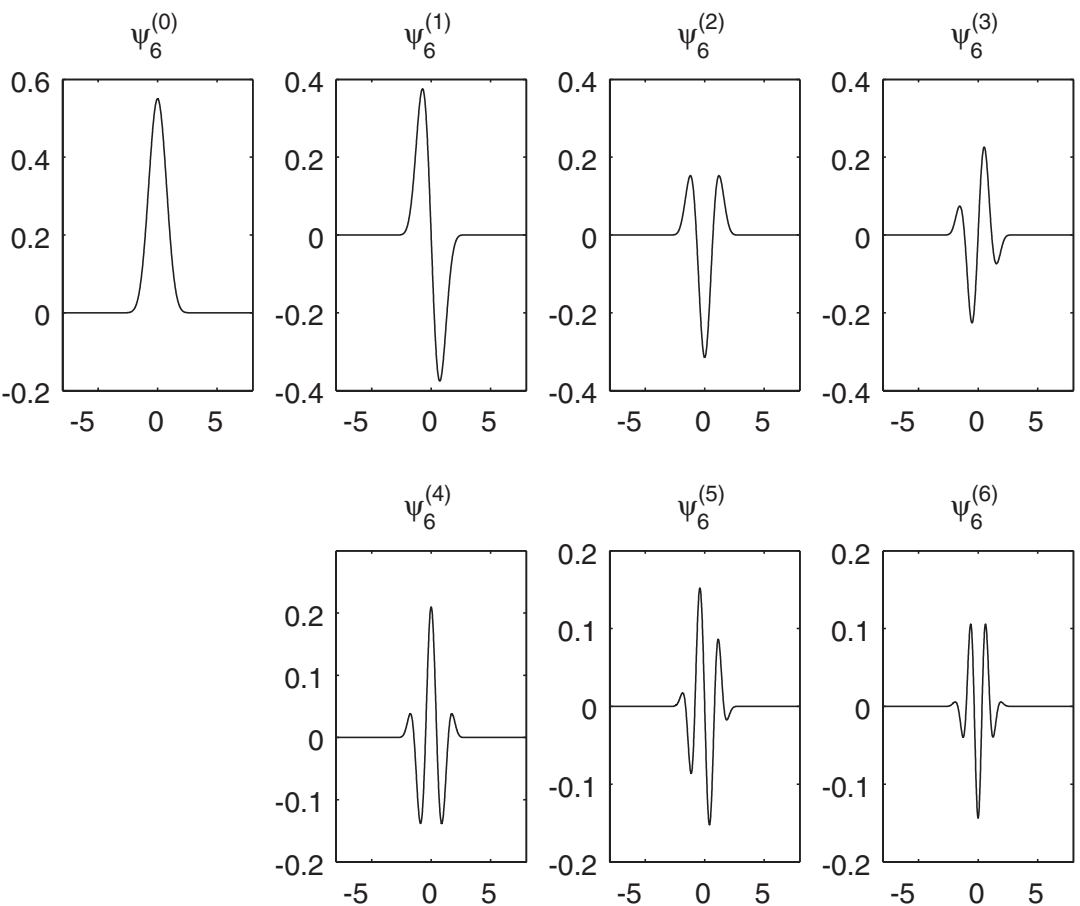

Fig. 14. Compare these graphs with Fig. 15. The function $\psi_{6}^{(j)}$ seems to have the same shape of the $j$ th derivative of the Gaussian function, however $\psi_{6}^{(j)}$ is compactly supported unlike Gaussian derivatives. 

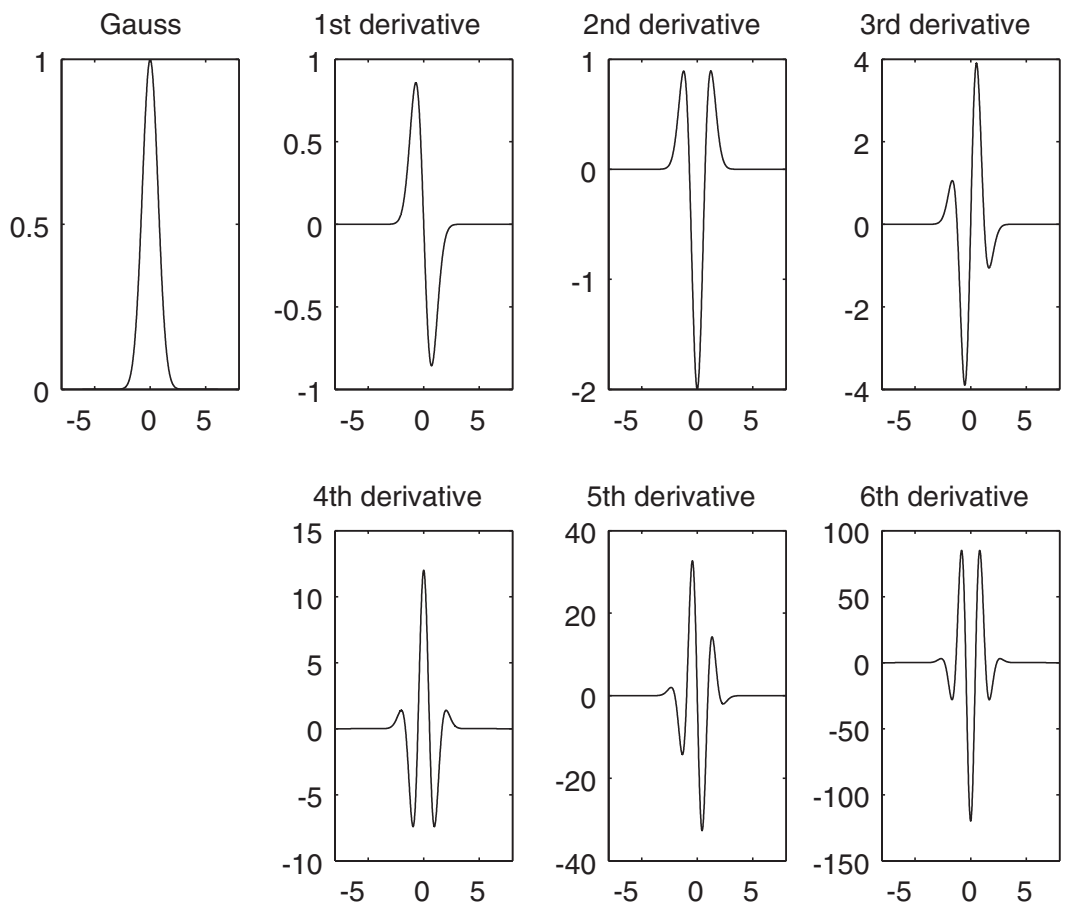

Fig. 15. These are graphs of derivatives of the Gaussian function. Here " $j$ th derivative" means $j$ th derivative of the Gaussian function.

decomposition of a signal can maintain its energy at a certain rate. This property is very convenient for achieving PR. In general, it is a difficult problem to find explicitly a synthesis bank. However, if a frame is tight, then the analysis filters carry out also the role of synthesis filters. Our first conclusion is that Gaussian-derivative-like filters of lower order gain the energy preserving property by cooperating with ones of higher order.

Now a crucial question arises: why do we need to take $m$ large, although $g_{1}^{(0)}$ and $g_{1}^{(1)}$ form an FPRMO filterbank or a tight frame? Our answer is that our filterbanks need filters $g_{m}^{(0)}, \ldots, g_{m}^{(m)}$ with large $m$ in order to improve the performance of orientation selectivity. More specifically, in the case of our filterbank, the more the number $m$ is larger, the more the orientation selectivity is better improved (see Section 7); all members of $g_{m}^{(0)}, \ldots, g_{m}^{(m)}$ contribute as a whole the improvement. Also in the visual system, the narrow bandpass filters similar to Gaussian derivatives of higher order may perform the same role. This is our answer to the question, and is also the second conclusion to the issue given at the beginning of this paragraph. These first and second conclusions are new viewpoints to this issue.

By the way, in their article [27], E. Salinas and L. E. Abbott investigate amply Gabor filters, and conclude that the set of V1 simple cell receptive fields is not tight. However, although $g_{m}^{(0)}, \ldots, g_{m}^{(m)}$ form a tight frame, their conclusion does not conflict with our attempt of applying our filterbanks based on these filters to the study of vision. The reason is that we never consider applying our filterbank directly to vision. We intend to construct our models of vision by endowing the filterbanks with some nonlinear processing. Indeed real visual information processing is carried out not only by simple cells in V1, but also by horizontal connection of neurons, and the latter is global and nonlinear processing; the nonlinear processing will break tightness and even perfect reconstruction property. In fact, in the previous paper [1], we gave a mathematical formulation of such a global, nonlinear processing, and constructed a model by bringing the nonlinear effect to a maximal overlap, bi-orthogonal, separable wavelet filterbank. Further, using this model, we gave good computer simulations of occurrence of certain visual illusions. Producing visual illusions implies that our model has not the perfect reconstruction property.

Lastly we give an additional remark. Separable wavelets which we adopted in [1] have only three directions: horizontal, vertical and diagonal. Therefore, in order to study vision by mathematical method more precisely, we need to construct nonlinear models based on our 2D filterbanks with better orientation selectivity. We will study the generalization in our subsequent papers.

Why do we need PR?

In this paper we have made an effort to let the filterbank possess PR. However a counterargument comes to mind. Is it appropriate to use a perfect reconstruction filterbank, although human's visual system is not PR? First of all, as we have mentioned in the previous paragraph, we intend to construct nonlinear models of vision based on PR filterbank, and the models themselves will be never PR. Still we think it is important that a filterbank which is a foundation of a nonlinear model of vision has the perfect reconstruction property. Indeed if the filterbank being the base of model of the vision has not PR, even though we construct mathematical algorithm of nonlinear effects in the brain, one can not judge 
whether the algorithm is appropriate or not, because differences between input and output images may be due to the failure of the filterbank itself.

We have also to discuss the synthesis bank. Our filterbank as well as usual filterbanks for signal processing consists of two phases, the analysis bank and the synthesis bank. In [32], S. Zeki pointed out that the brain has no localized areas where subband signals produced by parallel processing of input images are reconstructed to integrated images. In our study, the synthesis bank is used only for the technical purpose of visualizing what happens by mathematical processing modeled after real visual information processing.

\section{Receptive field models by other methods}

We close this section with a simple remark on emergence of receptive fields. Olshausen and Field ([23]), Olshausen, Salle and Lewicki ([24]), and Kohnen ([17]) proposed mathematical models of emergence of receptive fields. Our 2D filters with orientation selectivity are very similar in shape and spectra to the resulting receptive fields by sparse learning or by ASSOM. We can see the similarity by comparing Fig. 7 and Fig. 8 in our paper with figures in [23], [24] and [17, Fig. 5. 23, Fig. 5. 24].

\section{Appendix : Some Mathematical Remarks}

In the previous sections we have dealt with periodic extended images when $N_{1}$ and $N_{2}$ are even. In this section we consider other cases. For a natural number $N$, let

$$
N^{\prime}= \begin{cases}N+1, & \text { if } N \text { is odd, } \\ N, & \text { if } N \text { is even. }\end{cases}
$$

Suppose $N_{1}$ and $N_{2}$ are natural numbers. For a signal $\mathbf{x} \in l\left(\boldsymbol{Z}_{N_{1}} \times \boldsymbol{Z}_{N_{2}}\right)$, let $x_{e}\left[N_{1}^{\prime}, N_{2}^{\prime}\right]=x\left[N_{1}, N_{2}\right]$,

$$
\begin{aligned}
& x_{e}\left[n_{1}, n_{2}\right]=x\left[n_{1}, n_{2}\right], \\
& x_{e}\left[N_{1}^{\prime}, n_{2}\right]=x\left[N_{1}, n_{2}\right], x_{e}\left[n_{1}, N_{2}^{\prime}\right]=x\left[n_{1}, N_{2}\right]
\end{aligned}
$$

for $n_{1} \in \boldsymbol{Z}_{N_{1}}$, and $n_{2} \in \boldsymbol{Z}_{N_{2}}$. Then $\mathbf{x}_{e} \in l\left(\boldsymbol{Z}_{N_{1}^{\prime}} \times \boldsymbol{Z}_{N_{2}^{\prime}}\right)$. Since $N_{1}^{\prime}$ and $N_{2}^{\prime}$ are even, we can apply our decomposition methods to $\mathbf{x}_{e}$, and we have

$$
\mathbf{x}_{e}=\widetilde{\mathbf{F}}_{J}(0,0)+\sum_{j=1}^{J} \sum_{\substack{\mu_{1}, \mu_{2}=0, \mu_{1} \mu_{2}=0 \\ \mu_{1}+\mu_{2} \neq 0}}^{m} \widetilde{\mathbf{F}}_{j}\left(\mu_{1}, \mu_{2}\right)+\sum_{j=1}^{J} \sum_{\substack{\mu_{1}, \mu_{2}=0 \\ \mu_{1} \mu_{2}>0}}^{m}\left(\widetilde{\mathbf{F}}_{j}\left(\mu_{1}, \mu_{2}, 0\right)+\widetilde{\mathbf{F}}_{j}\left(\mu_{1}, \mu_{2}, 1\right)\right) .
$$

For $\mathbf{y} \in l\left(\boldsymbol{Z}_{N_{1}^{\prime}} \times \boldsymbol{Z}_{N_{2}^{\prime}}\right)$, let

$$
\mathbf{y}_{r}=\left(y\left[n_{1}, n_{2}\right]\right)_{n_{1} \in \boldsymbol{Z}_{N_{1}}, n_{2} \in \boldsymbol{Z}_{N_{2}}} \in l\left(\boldsymbol{Z}_{N_{1}} \times \boldsymbol{Z}_{N_{2}}\right) .
$$

Then by (11), we have that

$$
\mathbf{x}=\left(\mathbf{x}_{e}\right)_{r}=\widetilde{\mathbf{F}}_{J}(0,0)_{r}+\sum_{j=1}^{J} \sum_{\substack{\mu_{1}, \mu_{2}=0, \mu_{1} \mu_{2}=0 \\ \mu_{1}+\mu_{2} \neq 0}}^{m} \widetilde{\mathbf{F}}_{j}\left(\mu_{1}, \mu_{2}\right)_{r}+\sum_{j=1}^{J} \sum_{\substack{\mu_{1}, \mu_{2}=0 \\ \mu_{1} \mu_{2}>0}}^{m}\left(\widetilde{\mathbf{F}}_{j}\left(\mu_{1}, \mu_{2}, a\right)_{r}+\widetilde{\mathbf{F}}_{j}\left(\mu_{1}, \mu_{2}, b\right)_{r}\right) .
$$

Now we are concerned with symmetric extension signals. Suppose $N_{1}$ and $N_{2}$ are even. For $\mathbf{x}=$ $\left(x\left[n_{1}, n_{2}\right]\right)_{n_{1} \in \boldsymbol{Z}_{N_{1}}, n_{2} \in \boldsymbol{Z}_{N_{2}}}$, let $\mathbf{x}_{s y m}=\left(x\left[n_{1}, n_{2}\right]\right)_{n_{1} \in \boldsymbol{Z}_{2 N_{1}}, n_{2} \in \boldsymbol{Z}_{2 N_{2}}}$. By applying our theory for $l\left(\boldsymbol{Z}_{2 N_{1}} \times \boldsymbol{Z}_{2 N_{2}}\right)$ to $\mathbf{x}_{s y m}$, we have

$$
\mathbf{x}_{s y m}=\widetilde{\mathbf{F}}_{J}(0,0)+\sum_{j=1}^{J} \sum_{\substack{\mu_{1}, \mu_{2}=0, \mu_{1} \mu_{2}=0 \\ \mu_{1}+\mu_{2} \neq 0}}^{m} \widetilde{\mathbf{F}}_{j}\left(\mu_{1}, \mu_{2}\right)+\sum_{j=1}^{J} \sum_{\substack{\mu_{1}, \mu_{2}=0 \\ \mu_{1} \mu_{2}>0}}^{m}\left(\widetilde{\mathbf{F}}_{j}\left(\mu_{1}, \mu_{2}, a\right)+\widetilde{\mathbf{F}}_{j}\left(\mu_{1}, \mu_{2}, b\right)\right) .
$$

Now for every $\mathbf{y}=\left(y\left[n_{1}, n_{2}\right]\right)_{n_{1} \in \boldsymbol{Z}_{2 N_{1}}, n_{2} \in \boldsymbol{Z}_{2 N_{2}}}$, we denote by $\mathbf{y}_{\text {half }}$ the restricted array $\left(y\left[n_{1}, n_{2}\right]\right)_{n_{1} \in \boldsymbol{Z}_{N_{1}}, n_{2} \in \boldsymbol{Z}_{N_{2}}}$. Then from (12) it follows that

$$
\begin{aligned}
\mathbf{x}= & \left(\mathbf{x}_{\text {sym }}\right)_{\text {half }}=\widetilde{\mathbf{F}}_{J}(0,0)_{\text {half }}+\sum_{j=1}^{J} \sum_{\substack{\mu_{1}, \mu_{2}=0, \mu_{1} \mu_{2}=0 \\
\mu_{1}+\mu_{2} \neq 0}}^{m} \widetilde{\mathbf{F}}_{j}\left(\mu_{1}, \mu_{2}\right)_{\text {half }} \\
& +\sum_{j=1}^{J} \sum_{\substack{\mu_{1}, \mu_{2}=0 \\
\mu_{1} \mu_{2}>0}}^{m}\left(\widetilde{\mathbf{F}}_{j}\left(\mu_{1}, \mu_{2}, a\right)_{\text {half }}+\widetilde{\mathbf{F}}_{j}\left(\mu_{1}, \mu_{2}, b\right)_{\text {half }}\right) .
\end{aligned}
$$




\section{REFERENCES}

[1] Arai, H., "A nonlinear model of visual information processing based on discrete maximal overlap wavelets," Interdisciplinary Information Sciences, 11: 177-190 (2005).

[2] Arai, H., "Achromatic and chromatic visual information processing and discrete wavelets," (invited paper), in "Frontiers of Computational Science" (Y. Kaneda et al. eds.), 83-89, Springer-Verlag, (2007).

[3] Blakemore, C., and Campbell, F. W., "On the existence of neurones in the human visual system selectively sensitive to the orientation and size of retinal images," J. Physiol., 203: 237-260 (1969).

[4] Bruce, V., Green, P. R., and Georgeson, M. A., Visual Perception, Physiology, Psychology and Ecology, Psychology Press, (2003).

[5] Candés, E. J., and Donoho, D. L., Curvelets - a surprisingly effective nonadaptive representation of objects with edges, in Curve and Surface Fitting (A. Cohen et al. eds.), Vanderbilt Univ. Press, (1999).

[6] Coifman, R. R., and Donoho, D. L., "Translation invariant de-noising," Lecture Notes in Statistics, 103: 125-150 (1995).

[7] Daubechies, I., Han, B., Ron, A., and Shen, Z., "Framelets: MRA-based construction of wavelet frames," Appl. Comput. Harmon. Anal., 14: 1-46 (2003).

[8] De Valois, R. L., Albrecht, D. G., and Thorell, L. G., "Spatial frequency selectivity of cells in macaque visual cortex," Vision Res., 22: 545-559 (1982).

[9] De Valois, R. L., Yund, E. W., and Hepler, N., "The orientation and direction selectivity of cells in macaque visual cortex," Vision Res., 22: 531-544 (1982).

[10] Do M. N., and Vetterli, M., “The contourlet transform: an efficient directional multiresolution image representation," IEEE Trans. Image Processing, 14: 2091-2106 (2005).

[11] Elder, J. H., and Zucker, S. W., "Local scale control for edge detection and blur estimation," IEEE Trans. Pattern Analysis, 20: 699-716 (1998).

[12] Gray, T. W., and Glynn, J., Exploring Mathematics with Mathematica, Dialogs concerning Computers and Mathematics, Addison-Wesley (1994).

[13] Hubel, D. H., and Wiesel, T. N., "Receptive fields of single neurones in the cat's striate cortex," J. Phisiol., 148: 574-591 (1959).

[14] Hubel, D. H., and Wiesel, T. N., "Receptive fields, binocular interaction and functional architecture in the cat's visual cortex," J. Physiol., 160: 106-154 (1962).

[15] Hubel, D. H., and Wiesel, T. N., "Receptive fields and functional architecture of monkey striate cortex," J. Phisiol., 195: 215-243 (1968).

[16] Kingsbury, N. G., "Image processing with complex wavelets,” Phil. Trans. Roy. Soc. London, A357: 2543-2560 (1999).

[17] Kohonen, T., Self-Organizing Maps, Springer-Verlag, (2001).

[18] Marr, D., and Hildreth, E., "Theory of edge detection," Proc. R. Soc. Lond., B207: 187-217 (1980).

[19] Marr, D., Vision, W. H. Freeman \& Co. (1982).

[20] Matsuo, T., Yoshida, Y., and Nakamori, N., "Construction of 2D wavelets with directionality," Electronics and Communications in Japan, Part 3, 88: 1-10 (2005).

[21] Nason, G. P., and Silverman, B. W., "The stationary wavelet transform and some statistical applications," Lecture Notes in Statistics, 103: 281-299 (1995).

[22] Nguyen, T. T., "Multiresolution direction filterbanks: theory, design, and applications," IEEE Trans. Signal Processing, 33: 3895-3905 (2005).

[23] Olshausen, B. A., and Field, D. J., "Emergence of simple-cell receptive field properties by learning a sparse code for natural images," Nature, 607-609 (1996).

[24] Olshausen, B. A., Salle, P., and Lewicki, M. S., "Learning sparse image codes using a wavelet pyramid architecture," Advances in Neural Information Processing Systems 13: 887-893 (2001).

[25] Percival, D. B., and Walden, A. T., Wavelet Methods for Time Series Analysis, Cambridge Univ. Press (2000).

[26] Ron, A., and Shen, Z., "Affine systems in $L_{2}\left(\mathbb{R}^{d}\right)$ : the analysis of the analysis operator," J. Funct. Anal., 148: 408-447 (1997).

[27] Salinas, E., and Abbott, L. F., "Do simple cells in primary visual cortex form a tight frame?," Neural Comp., 12: 313-335 (2000).

[28] Selesnick, I. W., and Abdelnour, A. F., "Symmetric wavelet tight frame with two generators," Appl. Comput. Harmon. Anal., 17: 211-225 (2004).

[29] Vandergheynst, P., "Directional dyadic wavelet transforms: design and algorithm," IEEE Trans. Image Processing, 11: 363372 (2002).

[30] Webster, M. A., and De Valois, R. L., "Relationship between spatial-frequency and orientation tuning of striate-cortex cells," J. Opt. Soc. Am., 2: 1124-1132 (1985).

[31] Young, R. A., Oh say, can you see? The physiology of vision, in "Human Vision, Visual Processing, and Digital Display II," SPIE, 92-123 (1991).

[32] Zeki, S., A Vision of the Brain, Blackwell Scientific Publ. (1993). 